\title{
Block Bootstrap HAC Robust Tests: The Sophistication of the Naive Bootstrap
}

\author{
Sílvia Gonçalves \\ Département de sciences économiques, CIREQ and CIRANO, Université de Montréal
}

and

Timothy J. Vogelsang*

Department of Economics, Michigan State University

January 25, 2010

\begin{abstract}
This paper studies the properties of naive block bootstrap tests that are scaled by zero frequency spectral density estimators (long run variance estimators). The naive bootstrap is a bootstrap where the formula used in the bootstrap world to compute standard errors is the same as the formula used on the original data. Simulation evidence shows that the naive bootstrap can be much more accurate than the standard normal approximation. The larger the HAC bandwidth, the greater the improvement. This improvement holds for a large number of popular kernels, including the Bartlett kernel, and it holds when the i.i.d. bootstrap is used and yet the data are serially correlated. Using recently developed fixed- $b$ asymptotics for HAC robust tests, we provide theoretical results that can explain the finite sample patterns. We show that the block bootstrap, including the special case of the i.i.d. bootstrap, has the same limiting distribution as the fixed- $b$ asymptotic distribution. For the special case of a location model, we provide theoretical results that suggest the naive bootstrap can be more accurate than the standard normal approximation depending on the choice of the bandwidth and the number of finite moments in the data. Our theoretical results lay the foundation for a bootstrap asymptotic theory that is an alternative to the traditional approach based on Edgeworth expansions.
\end{abstract}

\footnotetext{
${ }^{*}$ For helpful comments and suggestions we thank an editor and two anonymous referees, Lutz Kilian, Guido Kuersteiner, Nour Meddahi, Ulrich Mueller, Pierre Perron, Yixiao Sun, Hal White, and seminar participants at Boston University, Queen's University, U. Toronto, U. of Western Ontario, Johns Hopkins Biostatistics, Chicago GSB, UCLA, UCSD, U. Michigan, U. Laval, U. Pittsburgh, U. Wisconsin, Cornell U., U. of Nottingham, ISEG, Banco de Portugal, the 2007 European Meetings of the Econometric Society in Budapest, the 2005 Winter Meetings of the Econometrics Society in Philadelphia, the 2005 European Economic Association Meetings in Amsterdam and the 2004 Forecasting Conference at Duke University. Vogelsang acknowledges financial support from the NSF through grant SES-0525707 whereas Gonçalves acknowledges financial support from the SSHRCC.
} 


\section{Introduction}

In this paper we analyze bootstrap procedures applied to tests based on heteroskedasticity autocorrelation (HAC) robust variance estimators using time series data that is covariance stationary. Because of dependence in the data, implementation of the bootstrap is more complicated than in the i.i.d. case. Many variants of the bootstrap have been proposed for dependent data including the well known moving blocks bootstrap originally proposed by Kunsch (1989). Theoretical conditions under which the block bootstrap can be expected to provide refinements have been established by Götze and Künsch (1996), Lahiri (1996), Andrews (2002) and others. Refinements of the block bootstrap in generalized method of moments (GMM) models have been shown by Hall and Horowitz (1996) and Inoue and Shintani (2006). The theoretical results in these papers have been established using Edgeworth expansions with leading terms that are distributed standard normal.

When the moving blocks bootstrap (MBB) is applied to tests based on heteroskedasticity autocorrelation robust variance estimators, a particular version of the MBB has been labeled "naive" by Davison and Hall (1993). The naive bootstrap uses the same formula for the HAC estimator in the bootstrap world as is used on the original data. While this may seem to be a natural way to proceed with the MBB, Davison and Hall (1993) and Götze and Künsch (1996) have shown that the naive MBB will not provide higher order accuracy as measured by Edgeworth expansions. To obtain higher order Edgeworth results they show that the HAC estimator in the bootstrap world needs to be computed using a formula that reflects the constraint on the correlation structure of the bootstrap data imposed by the moving blocks scheme. Recent work by Inoue and Shintani (2006) extends the Edgeworth analysis to certain testing problems in the GMM framework.

In a recent paper, Kiefer and Vogelsang (2005) reported small sample simulation results for HAC robust $t$-statistics for testing hypotheses about the sample mean of a stationary univariate time series. They found that the naive bootstrap, including the i.i.d. bootstrap, can dramatically outperform the standard normal approximation, and this improvement over the standard normal approximation occurs for many kernels including the Bartlett kernel. The case of the Bartlett kernel is interesting because the Edgeworth expansion results in the literature suggest that even the nonnaive version of the MBB will not be more accurate than the standard normal approximation in the Bartlett kernel case. The simulations reported by Kiefer and Vogelsang (2005) also exhibited an interesting and persistent pattern: the naive MBB, especially the i.i.d. bootstrap, closely mimics rejections that are obtained when using the fixed- $b$ asymptotic approximation proposed in their paper. Because these finite sample patterns are not predicted by the existing Edgeworth theory, an alternative theory is needed to understand the finite sample performance of the naive MBB.

In this paper we develop a theoretical framework that can be used to explain the finite sample 
patterns reported by Kiefer and Vogelsang (2005). We make two theoretical contributions. First, we provide sufficient conditions under which the naive MBB has the same first order fixed- $b$ asymptotic distribution as the original statistic. This result holds for fixed block lengths (including the special case of the i.i.d. bootstrap) and for block lengths that increase with the sample size but at a slower rate $^{1}$. This result explains why rejections using the naive MBB closely follow the rejections using fixed- $b$ asymptotic critical values. Second, in a simple location model and for the special case of the Bartlett kernel, we develop a higher order asymptotic theory to show that the i.i.d. bootstrap has an error in rejection probability (ERP) that may converge to zero faster than the ERP of the standard normal approximation depending on the bandwidth choice and the number of finite moments that exist in the data. Ex ante, it is not intuitively obvious that the i.i.d. bootstrap could be more accurate than the standard normal approximation (see Singh (1981)). Ex post, this property is no longer surprising when viewed from within the fixed- $b$ asymptotic framework.

In establishing the higher order properties of the i.i.d. bootstrap, we provide an upper bound on the error rate of the fixed- $b$ approximation and show that this is of the same order as the upper bound on the i.i.d. bootstrap error. Specifically, the bound we derive is of the order $O\left(T^{-1 / 2+3 /(2 p)}\right)$, where $p$ is the number of finite moments in the data. In contrast, the error rate of the normal approximation for one-tail tests based on the Bartlett kernel is $O\left(T^{-1 / 2}\right)+$ $O\left(\frac{M}{T}\right)+O\left(M^{-1}\right)$, where $M$ is the bandwidth parameter (see e.g. Sun and Phillips (2009)). Any rate for $M$ that is either larger or smaller than $O(\sqrt{T})$ implies a normal approximation error of magnitude larger than $O\left(T^{-1 / 2}\right)$. This is unequivocally larger than our upper bound when $p=\infty$; when $p<\infty$, it can be larger or smaller than our upper bound depending on the particular value of $p$ and how it relates to the rate of $M$. For instance, $p>9$ suffices if $M=O\left(T^{1 / 3}\right)$, which is the rate of the conventional MSE-optimal bandwidth parameter choice for the Bartlett kernel. If $M=O(\sqrt{T})$, which is the optimal-ERP bandwidth choice for one sided confidence intervals as recently found by Sun and Phillips (2009), the normal approximation error converges at the best possible rate of $O\left(T^{-1 / 2}\right)$. In this case, our upper bound on the fixed- $b$ (and the i.i.d. bootstrap) approximation is at least of the same order (they are of equal order only if $p=\infty$ as when the data is Gaussian) and our upper bound becomes uninformative.

Recently, Jansson (2004) and Sun, Phillips and Jin (2008) analyzed the higher order asymptotic properties of the fixed- $b$ asymptotic theory for the simple location model when the data is Gaussian. The upper bound on the error rate of the fixed- $b$ asymptotics we derive here when $p=\infty$ is not as fast as the rates found by Jansson (2004) and Sun et al. (2008). Thus, the bound on the ERP we provide is not sharp. While it is possible that the results in Jansson (2004) and Sun et al. (2008) extend to the non-Gaussian case studied here, establishing such results appear very difficult. Because Jansson (2004) and Sun et al. (2008) obtain results under the assumption that the data is Gaussian, their results cannot be applied to the bootstrap since the bootstrap data 
cannot be Gaussian by construction.

The remainder of the paper is organized as follows. In the next section we describe the model and test statistics. We review the fixed- $b$ asymptotic approximation. Section 3 reports simulation results for the simple location model and for a stationary regression model. The simulations illustrate the performance of the naive MBB relative to the standard normal and fixed- $b$ approximations. In Sections 4 and 5 we provide theoretical explanations for several of the patterns that emerge from the simulations. Section 4 focuses on stationary regression models and establishes the first order asymptotic equivalence between the naive bootstrap and the fixed- $b$ asymptotic approximation. These results could be generalized in straightforward ways to nonlinear models estimated by generalized method of moments. In Section 5 we narrow the focus to the simple location model and we provide higher order asymptotic results for Bartlett kernel based tests. These results establish that the fixed- $b$ asymptotic approximation and the naive i.i.d. bootstrap have ERPs that converge to zero at rates faster than the standard normal approximation. In Section 6 we discuss heuristic comparisons between fixed- $b$ asymptotic approximations and the Edgeworth approximations derived by Velasco and Robinson (2001) in an effort to shed some light on the relative performance of Edgeworth approximations and the naive bootstrap/fixed- $b$ asymptotics in the simple location model. Proofs are given in two mathematical appendices.

\section{$2 \quad$ Model and Test Statistics}

Throughout the paper we focus on stationary regression models of the form

$$
y_{t}=x_{t}^{\prime} \beta+u_{t}, \quad t=1,2, \ldots, T
$$

where $x_{t}$ and $\beta$ are $s \times 1$ vectors. The stationary time series $\left\{x_{t}\right\}$ and $\left\{u_{t}\right\}$ are autocorrelated and possibly conditionally heteroskedastic. It is assumed that $u_{t}$ is mean zero and is uncorrelated with $x_{t}$.

The parameter of interest is $\beta$ and its estimator is $\hat{\beta}=\left(\sum_{t=1}^{T} x_{t} x_{t}^{\prime}\right)^{-1} \sum_{t=1}^{T} x_{t} y_{t}$, the ordinary least squares (OLS) estimator. Let $Q=E\left(x_{t} x_{t}^{\prime}\right)$ and $\Omega=\lim _{T \rightarrow \infty} \operatorname{Var}\left(T^{-1 / 2} \sum_{t=1}^{T} v_{t}\right)$, where $v_{t}=x_{t} u_{t}$. For HAC robust testing we require estimates of $Q$ and $\Omega$. The usual estimate of $Q$ is $\hat{Q}=T^{-1} \sum_{t=1}^{T} x_{t} x_{t}^{\prime}$. Estimation of $\Omega$ is often implemented with a kernel variance estimator such as

$$
\hat{\Omega}=\sum_{j=-(T-1)}^{T-1} k\left(\frac{j}{M}\right) \hat{\Gamma}_{j},
$$

where $k(x)$ is a kernel function such that $k(x)=k(-x), k(0)=1,|k(x)| \leq 1, k(x)$ is continuous at $x=0$, and $\int_{-\infty}^{\infty} k^{2}(x)<\infty$. Here, for $j \geq 0, \hat{\Gamma}_{j}=T^{-1} \sum_{t=j+1}^{T} \hat{v}_{t} \hat{v}_{t-j}^{\prime}$ are the sample autocovariances of the score vector $\hat{v}_{t}=x_{t} \hat{u}_{t}$, with $\hat{u}_{t}=y_{t}-x_{t}^{\prime} \hat{\beta}$ the OLS residuals, and $\hat{\Gamma}_{j}=\hat{\Gamma}_{-j}^{\prime}$ for $j<0$. 
$M$ is the bandwidth parameter, which can act as a truncation lag for kernels such that $k(x)=0$ for $|x|>1$.

Consider testing the null hypothesis $H_{0}: R \beta=r$ against $H_{1}: R \beta \neq r$, where $R$ is a $q \times s$ matrix of rank $q$ and $r$ is a $q \times 1$ vector. We consider the following $F$-type statistic:

$$
F_{T}=T(R \hat{\beta}-r)^{\prime}\left[R \hat{Q}^{-1} \hat{\Omega} \hat{Q}^{-1} R^{\prime}\right]^{-1}(R \hat{\beta}-r) / q
$$

In the case where $q=1$ we can consider $t$-statistics of the form

$$
t_{T}=\frac{\sqrt{T}(R \hat{\beta}-r)}{\sqrt{R \hat{Q}^{-1} \hat{\Omega} \hat{Q}^{-1} R^{\prime}}} .
$$

Under suitable regularity conditions (described subsequently), $\sqrt{T}(R \hat{\beta}-r)$ can be approximated by a vector of normal random variables with variance-covariance matrix $R Q^{-1} \Omega Q^{-1} R^{\prime}$. Given that $p \lim \hat{Q}=Q$, the traditional asymptotic approach seeks to establish consistency of $\hat{\Omega}$ to justify approximating $\hat{\Omega}$ by $\Omega$. Consistency of $\hat{\Omega}$ requires that $M \rightarrow \infty$ as $T \rightarrow \infty$, but $M / T \rightarrow 0$. Under the traditional approach, $F_{T}$ has a limiting chi-square distribution and $t_{T}$ has a limiting standard normal distribution.

An alternative approximation for $\hat{\Omega}$ has been proposed by Kiefer and Vogelsang (2005). Suppose the bandwidth is modelled as $M=b T$, with $b$ a fixed constant in $(0,1]$. Because $b$ is held fixed in this asymptotic nesting of $M$, this approach has been labelled fixed- $b$ asymptotics. Under fixed- $b$ asymptotics, $\hat{\Omega}$ converges to a random variable (rather than a constant) that depends on the kernel and bandwidth. As a consequence, $F_{T}$ and $t_{T}$ have nonstandard distributions. These limiting distributions are useful for testing because they reflect the choice of bandwidth and kernel but are otherwise asymptotically pivotal (i.e. independent of nuisance parameters) and critical values can be tabulated. For example, under suitable regularity conditions (to be described subsequently), Kiefer and Vogelsang (2005) showed that

$$
\begin{aligned}
F_{T} & \Rightarrow W_{q}(1)^{\prime} Q_{q}(b)^{-1} W_{q}(1) / q, \\
t_{T} & \Rightarrow \frac{W_{1}(1)}{\sqrt{Q_{1}(b)}},
\end{aligned}
$$

where $\Rightarrow$ denotes weak convergence, $W_{i}(r)$ is an $i \times 1$ vector of independent standard Wiener processes and $Q_{i}(b)$ is a random matrix that depends on the kernel. For example, in the case of the Bartlett kernel,

$$
Q_{i}(b)=\frac{2}{b} \int_{0}^{1} \widetilde{W}_{i}(r) \widetilde{W}_{i}(r)^{\prime} d r-\frac{1}{b} \int_{0}^{1-b}\left(\widetilde{W}_{i}(r+b) \widetilde{W}_{i}(r)^{\prime}+\widetilde{W}_{i}(r) \widetilde{W}_{i}(r+b)^{\prime}\right) d r
$$

where $\widetilde{W}_{i}(r)=W_{i}(r)-r W_{i}(1)$. 
An alternative to asymptotic approximations is the bootstrap. In this paper we focus on the MBB of Kunsch (1989) and Liu and Singh (1992). Define the vector $w_{t}=\left(y_{t}, x_{t}^{\prime}\right)^{\prime}$ that collects the dependent and the explanatory variables for each observation. Let $\ell \in \mathbb{N}(1 \leq \ell<T)$ be a block length, and let $B_{t, \ell}=\left\{w_{t}, w_{t+1}, \ldots, w_{t+\ell-1}\right\}$ be the block of $\ell$ consecutive observations starting at $w_{t}$. Note that $\ell=1$ gives the standard i.i.d. bootstrap. For simplicity take $T=k_{0} \ell$. The MBB draws $k_{0}=T / \ell$ blocks randomly with replacement from the set of overlapping blocks $\left\{B_{1, \ell}, \ldots, B_{T-\ell+1, \ell}\right\}$. Let $F_{T}^{*}$ and $t_{T}^{*}$ denote the naive bootstrap versions of $F_{T}$ and $t_{T}$. $F_{T}^{*}$ and $t_{T}^{*}$ are computed as follows. Given a bootstrap resample $w_{t}^{*}=\left(y_{t}^{*}, x_{t}^{* \prime}\right)^{\prime}$, let $\widehat{\beta}^{*}$ denote the OLS estimate from the regression of $y_{t}^{*}$ on $x_{t}^{*}$ and let $\widehat{Q}^{*}=T^{-1} \sum_{t=1}^{T} x_{t}^{*} x_{t}^{* \prime}$. Let $\widehat{\Omega}^{*}$ denote the bootstrap version of $\widehat{\Omega}$ where $\hat{v}_{t}^{*}=x_{t}^{*} \hat{u}_{t}^{*}=x_{t}^{*}\left(y_{t}^{*}-x_{t}^{* \prime} \hat{\beta}^{*}\right)$ is used in place of $\widehat{v}_{t}$. The naive bootstrap statistics are defined as

$$
F_{T}^{*}=T\left(R \hat{\beta}^{*}-r^{*}\right)^{\prime}\left[R \hat{Q}^{*-1} \hat{\Omega}^{*} \hat{Q}^{*-1} R^{\prime}\right]^{-1}\left(R \hat{\beta}^{*}-r^{*}\right) / q
$$

where $r^{*}=R \widehat{\beta}$, and in the case of $q=1$,

$$
t_{T}^{*}=\frac{\sqrt{T}\left(R \hat{\beta}^{*}-r^{*}\right)}{\sqrt{R \hat{Q}^{*-1} \hat{\Omega}^{*} \hat{Q}^{*-1} R^{\prime}}} .
$$

Although the bootstrap statistics obviously depend on $\ell$, we do not index them by $\ell$ to simplify notation. These bootstrap statistics are naive in the sense that they are computed with standard errors that are of the same form as those used in computing $F_{T}$ and $t_{T}$ using the resampled data in place of the original data. The empirical distributions of $F_{T}^{*}$ and $t_{T}^{*}$ can be accurately estimated using simulations.

As a benchmark for the simulations in the next section, we also consider the version of the MBB proposed by Götze and Künsch (1996) which we label the GK-MBB. The GK bootstrap statistics take the same form as in (5) and (6) except that $r^{*}$ is replaced by

$$
r_{G K}^{*}=R \widetilde{\beta},
$$

where

$$
\begin{aligned}
\tilde{\beta} & =\left[E^{*}\left(\sum_{t=1}^{T} x_{t}^{*} x_{t}^{* \prime}\right)\right]^{-1} E^{*}\left(\sum_{t=1}^{T} x_{t}^{*} y_{t}^{*}\right) \\
& =\left(\frac{1}{T-\ell+1} \sum_{t=1}^{T-\ell+1} \frac{1}{\ell} \sum_{s=0}^{\ell-1} x_{t+s} x_{t+s}^{\prime}\right)^{-1} \frac{1}{T-\ell+1} \sum_{t=1}^{T-\ell+1} \frac{1}{\ell} \sum_{s=0}^{\ell-1} x_{t+s} y_{t+s} \\
& =\left(\sum_{t=1}^{T-\ell+1} \sum_{s=0}^{\ell-1} x_{t+s} x_{t+s}^{\prime}\right)^{-1} \sum_{t=1}^{T-\ell+1} \sum_{s=0}^{\ell-1} x_{t+s} y_{t+s},
\end{aligned}
$$


and $\hat{\Omega}^{*}$ is replaced with

$$
\tilde{\Omega}_{G K}^{*}=k_{0}^{-1} \sum_{i=1}^{k_{0}}\left(\ell^{-1 / 2} \sum_{j=1}^{\ell} \hat{v}_{(i-1) \ell+j}^{*}\right)\left(\ell^{-1 / 2} \sum_{t=1}^{\ell} \hat{v}_{(i-1) \ell+j}^{*}\right)^{\prime} .
$$

Note that $\tilde{\beta}$ and $\tilde{\Omega}_{G K}^{*}$ reflect the impact of the block structure of the resampled data on the mean and variance of $\hat{\beta}^{*}$. Recentering and rescaling with $r_{G K}^{*}$ and $\tilde{\Omega}_{G K}^{*}$ have the effect of making the Edgeworth expansions of the original and bootstrap statistics close to each other.

\section{$3 \quad$ Finite Sample Performance}

In this section we use simulations to compare and contrast the finite sample performance of the standard asymptotic approximation, the fixed- $b$ asymptotic approximation and the naive and GK bootstraps. We first present results for the simple location model followed by results for a stationary regression model with four regressors. The simple location model simulations illustrate interesting patterns in the relative performance of the different asymptotic approximations. Theoretical results that can explain some of these patterns are provided by the higher order asymptotic theory in Section 5. The simulations for the regression model show that the patterns seen in the simple location model continue to hold in the regression context. A theoretical link between the naive MBB and fixed- $b$ asymptotics suggested by both sets of simulations is formally established in Section 4 for regression models.

Consider the simple location model,

$$
y_{t}=\beta_{1}+u_{t},
$$

where

$$
u_{t}=\rho u_{t-1}+\left(1-\rho^{2}\right)^{1 / 2} \varepsilon_{t},
$$

$\left\{\varepsilon_{t}\right\} \sim$ i.i.d. $N(0,1)$ and $u_{1}=0$. We consider testing the null hypothesis that $\beta_{1} \leq 0$ against the alternative that $\beta_{1}>0$ at a nominal level of $5 \%$ using

$$
t_{\hat{\beta}_{1}}=\frac{\hat{\beta}_{1}}{\operatorname{se}\left(\hat{\beta}_{1}\right)}
$$

where se $\left(\hat{\beta}_{1}\right)$ is the HAC standard error estimate. We focus on a one-sided test because the theoretical results developed in Section 5 apply to one-sided tests. The true parameter, $\beta_{1}$, is set equal to zero and we consider three values for the $A R$ parameter $\rho: 0.0,0.5$ and 0.9 . In the simulations, 10,000 random samples are generated for the sample size $T=50$. Similar patters were found for other sample sizes. We consider the Bartlett and the QS kernels (results for other kernels are available from the authors) and report results across 25 different values of the bandwidth: 
$M=2,4, \ldots, 48,50$. We reject the null hypothesis whenever $t_{\hat{\beta}_{1}}>t_{c}$, where $t_{c}$ is a critical value.

The methods differ in the way in which the critical value is calculated. In particular, $t_{c}=1.645$ is used for the standard asymptotic approximation, whereas for the fixed- $b$ approximation $t_{c}$ is the $95 \%$ percentile of the fixed- $b$ asymptotic distribution derived by Kiefer and Vogelsang (2005). Given the sample size of 50, the values of $M$ implicitly determine the corresponding values of $b=0.02,0.04, \ldots, 0.98,1.0$ in the fixed- $b$ distribution theory. For the naive MBB and the GK-MBB $t_{c}$ is the $95 \%$ bootstrap percentile of the respective bootstrap $t$-statistics. The bootstrap tests are based on 999 replications for each sample. We report results for the block lengths $\ell=\{1,5\}$ where obviously $\ell=1$ is the i.i.d. bootstrap. Following a recommendation by Götze and Künsch (1996) we also implement both bootstraps using $\ell=M$.

We also report rejection probabilities using the Edgeworth approximation for $t_{c}$ derived by Velasco and Robinson (2001). Using $t_{z}$ to denote the right-tail $N(0,1)$ critical value, the Edgeworth critical value is given by

$$
t_{\text {edge }}=t_{z}+\frac{1}{2} \delta t_{z}^{2}+f\left(t_{z}\right) \frac{M}{T}
$$

where

$$
f(x)=\left(2 \int_{0}^{1} k(s)^{2} d s+\int_{0}^{1} k(s) d s\right) \frac{x}{2}+\left(\int_{0}^{1} k(s)^{2} d s\right)\left(\frac{x^{3}-3 x}{4}\right)
$$

and $\delta=M^{-1} \Omega^{-1} \sum_{j=-\infty}^{\infty}|j| \Gamma_{j}$ for the Bartlett kernel and $\delta=\frac{18}{125} \pi^{2} M^{-2} \Omega^{-1} \sum_{j=-\infty}^{\infty} j^{2} \Gamma_{j}$ for the QS kernel. Given the $A R(1)$ structure in the simulations, the formulas for $\delta$ simplify to $\frac{2 \rho}{M\left(1-\rho^{2}\right)}$ and $\frac{36 \pi^{2} \rho}{M^{2}(1-\rho)^{2}}$ respectively for the Bartlett and QS kernels ${ }^{2}$.

We implement the Edgeworth approximation in two ways. In the first, we make the unrealistic assumptions (from the perspective of practice) that it is known that the errors are $A R(1)$ and that the value of $\rho$ is known. This provides an infeasible benchmark. In the second, we replace $\Omega$ with $\widehat{\Omega}$ and we replace $\sum_{j=-\infty}^{\infty}|j| \Gamma_{j}$ and $\sum_{j=-\infty}^{\infty} j^{2} \Gamma_{j}$ with estimators $\sum_{j=-(T-1)}^{T-1} k\left(\frac{j}{M}\right)|j| \widehat{\Gamma}_{j}$ and $\sum_{j=-(T-1)}^{T-1} k\left(\frac{j}{M}\right) j^{2} \widehat{\Gamma}_{j}$ where $k(x)$ and $M$ are the same as used to construct $\widehat{\Omega}$. This feasible approach preserves the nonparametric nature of the test in that we are not assuming any knowledge about the form of the autocovariance structure.

Figures 1 and 2 contain results for the Bartlett kernel whereas Figures 3 and 4 contain results for the Quadratic Spectral (QS) kernel. Each figure contains three panels corresponding to the three values of $\rho$. Each panel depicts the empirical null rejection probabilities as a function of the bandwidth and implicitly as a function of $b=M / T$. Figures 1 and 3 focus on the naive MBB whereas Figures 2 and 4 provide comparisons between the naive MBB and the GK-MBB.

Looking at Figures 1 and 3 several interesting patterns emerge. The naive $\operatorname{MBB}(N B 1, N B 5)$ is 
(almost) always more accurate than the $N(0,1)$ asymptotic approximation and often substantially so. The improvement of the naive MBB over the $N(0,1)$ approximation holds for both kernels and the improvement is larger for the QS kernel as compared to the Bartlett kernel. The larger the bandwidth, the greater the improvement. The i.i.d. bootstrap (NB1) tends to closely follow the fixed- $b$ asymptotics across all DGP's, bandwidths, sample sizes and kernels, despite the presence of autocorrelation. This pattern strongly suggests a systematic relationship between the naive block bootstrap and the fixed- $b$ asymptotic approximation. In addition it is interesting to note that as $\rho$ increases (e.g. $\rho=0.9$ ), increasing the block size to 5 helps in further reducing the size distortions ${ }^{3}$. In this situation, ignoring the serial correlation in the model and using the i.i.d. bootstrap is inferior to using blocking. From a theoretical perspective this finite sample pattern suggests that the naive block bootstrap may offer an asymptotic refinement over the i.i.d. bootstrap/fixed-b asymptotics with careful choice of the block length. Such a theoretical investigation would be very difficult and is not considered here.

The performance of the Edgeworth approximations are interesting. The most relevant pattern is that neither Edgeworth approximation closely follows the naive MBB suggesting that theoretical explanations for the patterns displayed by the naive MBB will not be found using Edgeworth arguments. This is not surprising given the theoretical arguments made by Davison and Hall (1993) and Götze and Künsch (1996) about the naive MBB. When the errors are i.i.d. $(\rho=0)$, the infeasible Edgeworth (EdgeInf) is more accurate than the $N(0,1)$ approximation but is less accurate than the naive MBB or fixed- $b$ approximations. The differences become more apparent as the bandwidth increases. The feasible Edgeworth (EdgeFeas) gives rejections between the $N(0,1)$ and the infeasible Edgeworth. When there is serial correlation in the errors $(\rho=0.5,0.9)$, the feasible Edgeworth consistently is more accurate than the $N(0,1)$ but is less accurate than the naive MBB or fixed- $b$. The infeasible Edgeworth behaves much differently in the presence of serial correlation. When the bandwidth is small, the infeasible Edgeworth tends to under-reject, especially when $\rho=0.9$. When the bandwidth is large and the Bartlett kernel is used, the infeasible Edgeworth has rejections very similar to the naive MBB but it tends to over-reject more than those tests when the QS kernel is used. Overall it is interesting to note that the feasible and infeasible Edgeworth approximations do not seem systematically linked to each other.

Looking at Figures 2 and 4 it is apparent that the naive MBB and GK-MBB exhibit different patterns. When the block length is set to 5 , the naive bootstrap clearly follows the fixed- $b$ rejections whereas the GK bootstrap clearly follows the $N(0,1)$ rejections. Not surprisingly, the GK bootstrap reduces the over-rejection problem of the $N(0,1)$ approximation. Comparing the naive and GK bootstraps indicates that the naive bootstrap tends to deliver rejections closer to 0.05 than the GK bootstrap when $\ell=5$ for all three values of $\rho$, and as $M$ increases, the naive bootstrap has rejections much closer to 0.05 than the GK bootstrap. When setting $\ell=M$, both bootstraps 
perform reasonably well when $\ell$ and $M$ are small but they become erratic as $\ell$ and $M$ become large. This is to be expected given that theoretical results in the MBB literature (this paper included) require the block length to be of smaller order than the sample size.

To show that many of the patterns in the simple location model continue to hold in a regression setting, we now report results for a stationary regression model with four regressors using the well-studied setup of Andrews (1991). We consider the linear regression model

$$
y_{t}=\beta_{1}+\beta_{2} x_{t 2}+\beta_{3} x_{t 3}+\beta_{4} x_{t 4}+\beta_{5} x_{t 5}+u_{t}
$$

where $\left\{u_{t}\right\}$ is given by (8) and

$$
x_{t i}=\rho x_{t-1, i}+\left(1-\rho^{2}\right)^{1 / 2} v_{t i}, \quad x_{0 i}=0, \quad i=2, \ldots, 5 ;
$$

where $v_{t i}$ are generated as i.i.d. $N(0,1)$ errors that are independent of each other and independent with $u_{t}$. We used 2,000 replications.

We consider testing the null hypothesis that $\beta_{2}=0$ against the alternative that $\beta_{2} \neq 0$ at a nominal level of $5 \%$. The test statistic is

$$
t_{\hat{\beta}_{2}}=\frac{\hat{\beta}_{2}}{s e\left(\hat{\beta}_{2}\right)},
$$

where $s e\left(\hat{\beta}_{2}\right)$ is a HAC standard error estimate. Because $t_{\hat{\beta}_{2}}$ is exactly invariant to the values of the other regression parameters, we set them to zero without loss of generality. We reject the null hypothesis whenever $\left|t_{\hat{\beta}_{2}}\right|>t_{c}$, where $t_{c}$ is a critical value. We use the same setup as in the simple location model except that we report results for a wider range of block lengths $\ell \in\{1,5,10,25\}$. We do not report Edgeworth results because formal Edgeworth expansions do not appear available for regression models. We consider a two-sided test in the regression case to show that many of the patterns seen for the one-sided test in the simple location model carry over to two-sided tests.

Results for the naive bootstrap are given in Figures 5 and 7. As in the simple location model, we see that the i.i.d. bootstrap $(N B 1)$ closely follows the rejections of the fixed- $b$ approximation and both are usually more accurate than the $N(0,1)$ approximation. When the serial correlation in the errors is strong $(\rho=0.9)$ increasing the block length to 5 (NB5) and 10 (NB10) can further increase the accuracy of the approximation. Making the block length too big relative to the sample size and the strength of the serial correlation $(\rho=0,0.5)$ can result in substantial over-rejections and the naive bootstrap can be a worse approximation than the $N(0,1)$ when $M$ small to medium in size. In this regression setting we see that the choice of block length matters. Using a small block length, including the i.i.d. bootstrap, does show improvement over the $N(0,1)$ approximation and the link to the fixed- $b$ rejections is strong. But, it is clear that further improvements are possible by using a larger block length when the serial correlation is strong as long the block length isn't 
too large. Again we see that refinements over the fixed- $b$ approximation are potentially possible with the naive bootstrap with judicious choice of block length.

Figures 6 and 8 allow comparisons of the naive and GK bootstraps with $\ell=10$ and $\ell=M$. Focusing on the case of $\ell=10$, we see that the naive bootstrap performs better than both the $N(0,1)$ and fixed- $b$ approximations. As $M$ increases, rejections become close to 0.05 for both kernels and all three values of $\rho$. The GK bootstrap shows different patterns. It tends to under-reject with $M$ is small and tends to over-rejects when $M$ is large although is also performs better than both the $N(0,1)$ and fixed- $b$ approximations. With $\ell=M$ both bootstraps have similar rejections when $\ell, M$ are small and both are improvements over the asymptotic approximations. But, as $\ell, M$ increase, the similarity of the bootstraps begins to break down and both bootstraps become erratic as $\ell, M$ become large.

In unreported simulations we computed finite sample power ${ }^{4}$ of the tests over a grid of values for $\beta_{1}$ in the location model and $\beta_{2}$ in the regression model. We found that power of the naive bootstrap closely follows power when using the fixed- $b$ critical value. As $M$ increases, power of both the naive MBB and fixed- $b$ decrease and this pattern is consistent with local asymptotic power calculations reported by Kiefer and Vogelsang (2005). Power using the $N(0,1)$ approximation is often higher but this is largely due to the substantial over-rejection problem of the normal approximation under the null.

The patterns in the simulations in both the simple location and regression models suggest that the fixed- $b$ approximation and the naive MBB are systematically related and that they may provide an improvement over the $N(0,1)$ approximation. Careful choice of the block length may provide an improvement over the fixed- $b$ approximation. Comparisons to the GK-MBB suggest that the naive and GK bootstraps are not systematically related. Obtaining theoretical results that explain all of these patterns is a very challenging research program. In the next section we establish the asymptotic equivalence of the fixed- $b$ approximation and the naive MBB in stationary regression models. We then focus on the simple location model and show that the naive MBB theoretically is more accurate than the $N(0,1)$ approximation when the Bartlett kernel is used. The very difficult question as to whether with careful choice of the block length the naive bootstrap can improve upon the fixed- $b$ approximation is beyond the scope of this paper.

\section{Fixed- $b$ Bootstrap Asymptotics}

In this section we derive the asymptotic distribution of naive block bootstrap HAC robust tests under the fixed- $b$ asymptotics. In particular, for linear regression models we show that $t_{T}^{*}$ and $F_{T}^{*}$ have the same limiting fixed- $b$ distribution as $t_{T}$ and $F_{T}$. Define $S_{[r T]}=\sum_{t=1}^{[r T]} v_{t}$, where $[r T]$ denotes the integer part of $r T$ with $r \in[0,1]$. Let $X_{T}(r)=T^{-1 / 2} S_{[r T]}$ be the corresponding partial sum process. Similarly, define $Q_{T}(r)=T^{-1} \sum_{t=1}^{[r T]} x_{t} x_{t}^{\prime}$. Following Kiefer and Vogelsang (2005) we 
make the following two high level assumptions:

A1. $X_{T}(r) \Rightarrow \Lambda W_{s}(r)$, with $\Omega=\Lambda \Lambda^{\prime}=\lim _{T \rightarrow \infty} \operatorname{Var}\left(T^{-1 / 2} \sum_{t=1}^{T} v_{t}\right)$.

A2. $\sup _{r \in[0,1]}\left|Q_{T}(r)-r Q\right| \rightarrow 0$ in probability.

Here, we assume in addition that the statistic of interest, $A_{T}$, is such that:

A3. $A_{T}$ can be written as

$$
A_{T}=g\left(X_{T}(r), Q_{T}(r), D_{T}(r)\right)
$$

where $g$ is a continuous functional of $\left(X_{T}(r), Q_{T}(r), D_{T}(r)\right)$, and $D_{T}(r)$ is a vector of deterministic functions of $T$ and $r$ such that $D_{T}(r) \rightarrow D(r)$ as $T \rightarrow \infty$, uniformly in $r$.

Condition A3 is a general way of expressing statistics that includes $t_{T}$ and $F_{T}$. The function $D_{T}(r)$ reflects the choice of kernel. Using the arguments of Kiefer and Vogelsang (2005), it follows that $\hat{\Omega}$ is a continuous functional of the processes $X_{T}(r), Q_{T}(r)$, and $D_{T}(r)$, where $D_{T}(r)$ is a function of $k(r)$. If $k^{\prime \prime}(r)$ exists, then we can show that $\lim _{T \rightarrow \infty} D_{T}(r)=b^{-2} k^{\prime \prime}(r / b)$, in which case $D(r)=b^{-2} k^{\prime \prime}(r / b)$. For kernels that truncate to zero for $|x| \geq 1, D_{T}(r)$ is a $2 \times 1$ vector and $D(r)$ has elements given by $b^{-2} k^{\prime \prime}(r / b)$ for $|r| \leq b$ and $b^{-1} k_{-}^{\prime}(1)$, where $k_{-}^{\prime}(1)$ is the first derivative of $k(x)$ from the left evaluated at $x=1$. For the Bartlett kernel we have $D_{T}(r)=b$ and $D(r)=b$. Thus, A3 holds for a wide class of kernels including the Bartlett kernel. See Kiefer and Vogelsang (2005) for additional details on how $D_{T}(r)$ is constructed.

Under conditions A1 through A3, an application of the continuous mapping theorem (CMT) implies that as $T \rightarrow \infty$,

$$
A_{T} \Rightarrow g\left(\Lambda W_{s}(r), r Q, D(r)\right) \equiv G .
$$

Suppose that the random variable $G$ is pivotal, i.e. its distribution is invariant to $\Lambda$ and $Q$. For example, this is the case for $F_{T}$ and $t_{T}$ as indicated by (3). The goal in this section is to provide a set of primitive conditions on $\left\{x_{t}\right\}$ and $\left\{v_{t}\right\}$ under which the naive block bootstrap test, $F_{T}^{*}$, weakly converges to $G$, in which case the naive bootstrap and the fixed- $b$ approximation will be equivalent in a first order sense. Note that results for $t_{T}^{*}$ follow as an obvious corollary.

We now need to introduce some additional notation. Given a bootstrap resample $\left\{w_{t}^{*}=\left(y_{t}^{*}, x_{t}^{* \prime}\right)^{\prime}\right\}$, let $v_{0 t}^{*}=x_{t}^{*}\left(y_{t}^{*}-x_{t}^{* \prime} \beta\right) \equiv x_{t}^{*} u_{0 t}^{*}$, and let $v_{t}^{*}=x_{t}^{*}\left(y_{t}^{*}-x_{t}^{* \prime} \hat{\beta}\right)^{\prime} \equiv x_{t}^{*} u_{t}^{*}$. In order to simplify the notation, we omit $T$ in the definition of the bootstrap variables, e.g., we write $v_{t}^{*}$ instead of $v_{T t}^{*}$. Notice that $v_{t}^{*}$ (and not $v_{0 t}^{*}$ ) is the bootstrap analogue of $v_{t}$ as it replaces $\beta$ with $\hat{\beta}$. Let $S_{[r T]}^{*}=\sum_{t=1}^{[r T]} v_{t}^{*}$ and define the bootstrap partial sum process $X_{T}^{*}(r)=T^{-1 / 2} S_{[r T]}^{*}$. Similarly, define $Q_{T}^{*}(r)=T^{-1} \sum_{t=1}^{[r T]} x_{t}^{*} x_{t}^{* \prime}$. As usual in the bootstrap literature, $P^{*}$ denotes the probability measure induced by the bootstrap resampling, conditional on a realization of the original time 
series. We use the following notation for the bootstrap asymptotics (see Chang and Park (2003) for similar notation and for several useful bootstrap asymptotic properties): Let $Z_{T}^{*}$ be a sequence of bootstrap statistics. We write $Z_{T}^{*}=o_{P^{*}}(1)$ in probability, or $Z_{T}^{*} \rightarrow^{P^{*}} 0$ in probability, if for any $\varepsilon>0, \delta>0, \lim _{T \rightarrow \infty} P\left[P^{*}\left(\left|Z_{T}^{*}\right|>\delta\right)>\varepsilon\right]=0$. Similarly, we write $Z_{T}^{*}=O_{P^{*}}(1)$ in probability if for all $\varepsilon>0$ there exists a $M_{\varepsilon}<\infty$ such that $\lim _{T \rightarrow \infty} P\left[P^{*}\left(\left|Z_{T}^{*}\right|>M_{\varepsilon}\right)>\varepsilon\right]=0$. Finally, we write $Z_{T}^{*} \Rightarrow^{P^{*}} Z$ in probability if, conditional on the sample, $Z_{T}^{*}$ weakly converges to $Z$ under $P^{*}$, for all samples contained in a set with probability converging to one. Specifically, we write $Z_{T}^{*} \Rightarrow^{P^{*}} Z$ in probability if and only if $E^{*}\left(f\left(Z_{T}^{*}\right)\right) \rightarrow E(f(Z))$ in probability for any bounded and uniformly continuous function $f$.

Suppose the bootstrap processes $X_{T}^{*}(r)$ and $Q_{T}^{*}(r)$ satisfy the following assumptions, in probability:

$\mathbf{A 1}{ }^{*} . X_{T}^{*}(r) \Rightarrow^{P^{*}} \Lambda^{*} W_{s}(r)$, for some $\Lambda^{*}$

$\mathbf{A 2}{ }^{*} \sup _{r \in[0,1]}\left|Q_{T}^{*}(r)-r Q^{*}\right| \rightarrow^{P^{*}} 0$ for some $Q^{*}$.

In this section we study the asymptotic behavior of naive bootstrap statistics, i.e., we suppose that

A3* $^{*}$ The bootstrap statistic $A_{T}^{*}$ can be written as

$$
A_{T}^{*}=g\left(X_{T}^{*}(r), Q_{T}^{*}(r), D_{T}(r)\right)
$$

where $g$ and $D_{T}(r)$ are as defined in A3.

According to condition $\mathrm{A} 3^{*}$, the bootstrap statistic is equal to the exact same function as the original statistic, but replaces the bootstrap data for the real data. This is the sense in which the bootstrap statistic is naive. It is a very straightforward algebraic calculation to show that $t_{T}^{*}$ and $F_{T}^{*}$ satisfy condition $\mathrm{A} 3^{*}$. In particular, note that the recentering of $\hat{\beta}^{*}$ around $\hat{\beta}$ ensures that the bootstrap statistics can be expressed as the same functionals of $X_{T}^{*}(r), Q_{T}^{*}(r)$ and $D_{T}(r)$ as the original statistics. It is clear that under Assumptions $\mathrm{A} 1^{*}-\mathrm{A} 3^{*}$, by an application of the CMT, we have that

$$
A_{T}^{*} \Rightarrow^{P^{*}} g\left(\Lambda^{*} W_{s}(r), r Q^{*}, D(r)\right),
$$

in probability. Because the random variable $g(\cdot, \cdot, \cdot)$ is pivotal (as in the case of $t$ and $F$ tests), the limiting distribution of $A_{T}^{*}$ coincides with the limiting distribution of $A_{T}$, independently of $\Lambda^{*}$ and $Q^{*}$. Thus, the asymptotic equivalence between $A_{T}^{*}$ and $A_{T}$ depends crucially on the conditions $\mathrm{A} 1^{*}$ and $\mathrm{A} 2^{*}$. Next, we provide primitive conditions on $\left\{x_{t}\right\}$ and $\left\{v_{t}\right\}$ that are sufficient for $\mathrm{A} 1^{*}$ and $\mathrm{A} 2^{*}$. 
We derive results under the assumptions that $\left\{x_{t}\right\}$ and $\left\{v_{t}\right\}$ are near epoch dependent (NED) on an underlying mixing process $\left\{\varepsilon_{t}\right\}$. NED processes allow for very general forms of dependence and contain mixing processes as a special case. For a general time series $\left\{w_{t}\right\}$, we view each coordinate $w_{t}$ as a measurable function of the potentially infinite history of another underlying process $\left\{\varepsilon_{t}\right\}$, i.e. $w_{t}\left(\ldots, \varepsilon_{t-1}, \varepsilon_{t}, \varepsilon_{t+1}, \ldots\right)$. Let $\mathcal{F}_{s}^{t} \equiv \sigma\left(\varepsilon_{s}, \ldots, \varepsilon_{t}\right)$ for any $s \leq t$ be the sigma-field generated by $\varepsilon_{s}, \ldots, \varepsilon_{t}$, and let $E_{s}^{t}$ denote the expectation conditional on $\mathcal{F}_{s}^{t}$. We say $\left\{w_{t}\right\}$ is $L_{q}$-NED on $\left\{\varepsilon_{t}\right\}, q \geq$ 1 , if $\left\|w_{t}\right\|_{q}<\infty$ and $\nu_{k_{0}}=\sup _{t}\left\|w_{t}-E_{t-k_{0}}^{t+k_{0}} w_{t}\right\|_{q} \rightarrow 0$ as $k_{0} \rightarrow \infty$. Here and in what follows, $\|w\|_{q}=$ $\left(\sum_{i} E\left|w_{i}\right|^{q}\right)^{1 / q}$ denotes the $L_{q}$-norm of a random vector $w$. Similarly, we let $|\cdot|$ denote the Euclidean norm of the corresponding vector or matrix. If the NED coefficients $\nu_{k_{0}}$ are such that $\nu_{k_{0}}=$ $O\left(k_{0}^{-a-\delta}\right)$ for some $\delta>0$, we say $\left\{w_{t}\right\}$ is $L_{q}$-NED of size $-a$. We assume $\left\{\varepsilon_{t}\right\}$ is strong mixing. The strong mixing coefficients are $\alpha_{k_{0}}=\sup _{m} \sup _{\left\{A \in \mathcal{F}_{-\infty}^{m}, B \in \mathcal{F}_{m+k_{0}}^{\infty}\right\}}|P(A \cap B)-P(A) P(B)|$; we require $\alpha_{k_{0}} \rightarrow 0$ as $k_{0} \rightarrow \infty$ suitably fast.

We impose the following assumptions on $\left\{x_{t}\right\}$ and $\left\{v_{t}\right\}$ :

\section{Assumption 1}

1a. For some $p>2,\left\|x_{t}\right\|_{2 p} \leq \Delta<\infty$ for all $t=1,2, \ldots$.

1b. $\left\{x_{t}\right\}$ is a weakly stationary sequence $L_{2}$-NED on $\left\{\varepsilon_{t}\right\}$ with NED coefficients of size $-\frac{2(p-1)}{p-2}$.

1c. $\left\|v_{t}\right\|_{p} \leq \Delta<\infty$, and $E\left(v_{t}\right)=0$ for all $t=1,2, \ldots$.

1d. $\left\{v_{t}\right\}$ is a weakly stationary sequence $L_{2}$-NED on $\left\{\varepsilon_{t}\right\}$ with NED coefficients of size $-\frac{1}{2}$.

1e. $\left\{\varepsilon_{t}\right\}$ is an $\alpha$-mixing sequence with $\alpha_{k_{0}}$ of size $-\frac{2 p}{p-2}$.

1f. $\Omega=\lim _{T \rightarrow \infty} \operatorname{Var}\left(T^{-1 / 2} \sum_{t=1}^{T} v_{t}\right)$ is positive definite.

We can show that Assumption 1 is a sufficient assumption for the high level conditions A1 and A2. Note that under Assumption $1, \Omega=\lim _{T \rightarrow \infty} \operatorname{Var}\left(T^{-1 / 2} S_{T}\right)$ exists. We further assume $\Omega$ is positive definite, which ensures the existence of a matrix $\Lambda$ such that $\Omega=\Lambda \Lambda^{\prime}$. Next, we show that the following strengthened version of Assumption 1 is sufficient to ensure that conditions $\mathrm{A} 1^{*}$ and $\mathrm{A} 2^{*}$ hold.

\section{Assumption $1^{\prime}$}

$\mathbf{1} \mathbf{c}^{\prime}$. For some $p>2$ and $\delta>0,\left\|v_{t}\right\|_{p+\delta} \leq \Delta<\infty$, and $E\left(v_{t}\right)=0$ for all $t=1,2, \ldots$.

$\mathbf{1 d}^{\prime} .\left\{v_{t}\right\}$ is a weakly stationary sequence $L_{2+\delta}$-NED on $\left\{\varepsilon_{t}\right\}$ with NED coefficients of size -1 .

1e' $\mathbf{e}^{\prime}\left\{\varepsilon_{t}\right\}$ is an $\alpha$-mixing sequence of size $-\frac{(2+\delta)(p+\delta)}{p-2}$. 
Lemma 4.1 Under Assumption 1 strengthened by Assumption 1', it follows that,

a) For any fixed $\ell$ such that $1 \leq \ell<T$, as $T \rightarrow \infty$,

$$
X_{T}^{*}(r) \Rightarrow^{P^{*}} \Lambda_{\ell} W_{s}(r)
$$

in probability, where $\Lambda_{\ell}$ is the square root matrix of $\Omega_{\ell} \equiv \Gamma_{0}+\sum_{j=1}^{\ell}\left(1-\frac{j}{\ell}\right)\left(\Gamma_{j}+\Gamma_{j}^{\prime}\right)$.

b) Let $\ell=\ell_{T} \rightarrow \infty$ as $T \rightarrow \infty$ such that $\ell^{2} / T \rightarrow 0$. Then

$$
X_{T}^{*}(r) \Rightarrow^{P^{*}} \Lambda W_{s}(r),
$$

in probability, where $\Lambda$ is the square root matrix of $\Omega$.

c) Under both sets of assumptions on $\ell$, it follows that

$$
\sup _{r \in[0,1]}\left|Q_{T}^{*}(r)-r Q\right| \rightarrow^{P^{*}} 0
$$

in probability.

Parts a) and b) of Lemma 4.1provide functional central limit theorems (FCLT) for the bootstrap partial sum process of the bootstrap scores $X_{T}^{*}(r)=T^{-1 / 2} \sum_{t=1}^{[r T]} v_{t}^{*}$. To prove these results, we apply a bootstrap FCLT (Lemma A.3 given in the Appendix) for $Z_{T}^{*}(r)=T^{-1 / 2} \sum_{t=1}^{[r T]}\left(X_{t}^{*}-E^{*}\left(X_{t}^{*}\right)\right.$ ), when $\left\{X_{t}^{*}\right\}$ is a MBB resample of $\left\{X_{t}\right\}$, a NED process on a mixing process. Lemma A.3 is a multivariate extension of an univariate bootstrap FCLT given in Paparoditis and Politis (2003) for stationary mixing processes to the NED case.

We consider two cases: a) one where $\ell$ is fixed as $T \rightarrow \infty$, and b) another where $\ell \rightarrow \infty$ as $T \rightarrow \infty$. Note that the first case includes the i.i.d. bootstrap as a special case. According to Lemma 4.1, the bootstrap partial sum process $X_{T}^{*}(r)$ weakly converges to a Brownian motion with the "right" covariance matrix $\Omega$ only if the block size $\ell$ increases with the sample size at an appropriate rate. When $\ell$ is fixed, the limiting covariance matrix is $\Omega_{\ell}$, which is different from $\Omega$ under general autocorrelation. This reflects the well-known fact that the MBB with fixed block size (and therefore the i.i.d. bootstrap) achieves only partial correction of dependence (cf. Liu and Singh (1992)).

Our first formal theoretical result is as follows.

Theorem 4.1 Let $b \in(0,1]$ be a constant and suppose $M=b T$. Let Assumption 1 strengthened by Assumption $1^{\prime}$ hold, and let $k(x)$ be the Bartlett kernel or let $k(x)$ be such that $k^{\prime \prime}(x)$ exists and is continuous everywhere with the possible exception of $|x|=1$. Suppose the block size $\ell$ is either fixed as $T \rightarrow \infty$, or $\ell \rightarrow \infty$ as $T \rightarrow \infty$ such that $\ell^{2} / T \rightarrow 0$. Then, under $H_{0}: R \beta=r$, as $T \rightarrow \infty$,

$$
F_{T}^{*} \Rightarrow^{P^{*}} W_{q}(1)^{\prime} Q_{q}(b)^{-1} W_{q}(1) / q,
$$


in probability, where $Q_{q}(b)$ is a random matrix defined in Definition 1 of Kiefer and Vogelsang (2005).

Theorem 4.1 shows that the naive bootstrap $F$ test statistic has asymptotically the same distribution of $F_{T}$ derived under the fixed- $b$ asymptotics nesting of Kiefer and Vogelsang (2005). A similar result holds for $t_{T}^{*}$. The first implication of Theorem 4.1 is that a naive bootstrap is as accurate to first order as the first order fixed- $b$ asymptotics of Kiefer and Vogelsang (2005). The second implication is that a simple i.i.d. bootstrap is asymptotically valid (and equivalent to first order to the fixed- $b$ asymptotic limit), even in the presence of serial correlation. This result is a consequence of the asymptotic pivotalness of the $F$ statistic.

\section{Higher-order results}

In this section we provide an upper bound on the rate of convergence of the error implicit in the naive i.i.d. bootstrap approximation and show that it can be smaller than the error of the $N(0,1)$ approximation even for dependent data. We focus on the $t$-statistic in the simple location model given by (7), i.e. we assume $x_{t} \equiv 1$ for all $t$. Here the score vector $v_{t}$ is equal to the scalar $u_{t}$. We

derive results for the Bartlett kernel because $\widehat{\Omega}$ can be expressed as a relatively simple function of $X_{T}(r)$ in this case. We expect our results to naturally extend to other kernels although the details are likely to be very tedious.

In this section we assume $u_{t}$ is a linear process. This is a more restrictive dependence assumption than our previous NED Assumption 1. To prove our results, we will rely on the method of strong approximations (see below for more details on this method), available for linear processes, and this is the main reason why we restrict attention to the special class of linear processes. We are unaware of such results for NED sequences. Thus, we let

$$
u_{t}=\pi(L) \varepsilon_{t}=\sum_{j=0}^{\infty} \pi_{j} \varepsilon_{t-j},
$$

with $\pi(z)=\sum_{j=0}^{\infty} \pi_{j} z^{j}$, and make the following additional assumptions.

\section{Assumption 2}

a) $\varepsilon_{t}$ are i.i.d. with $E\left(\varepsilon_{t}\right)=0, E\left(\varepsilon_{t}^{2}\right)=\sigma^{2}$ and $E\left|\varepsilon_{t}\right|^{p}<\infty$ for some $p>2$.

b) $\pi(z) \neq 0$ for all $|z| \leq 1$ and $\sum_{i=0}^{\infty}|i|\left|\pi_{i}\right|<\infty$.

Under Assumption 2, the FCLT for linear processes (cf. Theorem 3.4, Phillips and Solo, 1992) implies that

$$
W_{T}^{0}(r) \equiv T^{-1 / 2} \sum_{t=1}^{[T r]} u_{t} \Rightarrow \Lambda W_{1}(r)
$$


where $\Omega=\Lambda^{2} \equiv \pi^{2}(1) \sigma^{2}$ is the long run variance under Assumption 2. To establish our results we need a result stronger than this invariance principle. In particular, we need specific rates of convergence of the partial sum process for its limiting process. This can be achieved through the method of strong approximations. Recently, Park (2003) uses strong approximations to show that the bootstrap provides an asymptotic refinement for unit root tests. Similarly, Park (2006) relies on this method to show asymptotic refinements of the bootstrap in the context of weakly integrated processes. Our methods of proof will closely follow those of Park (2006).

Consider the following probabilistic embedding of the partial sum process of $u_{t}$ :

$$
W_{T}(r)={ }_{d} T^{-1 / 2} \sum_{t=1}^{[T r]} u_{t},
$$

where $={ }_{d}$ denotes equality in distribution. $W_{T}$ is a stochastic process on $D[0,1]$ having the same distribution as $W_{T}^{0}$. In what follows, we will not make a distinction between $W_{T}$ and its distributionally equivalent copy $W_{T}^{0}$. Therefore we will interpret the distributional equality $=_{d}$ as the usual equality. The Skohorod representation theorem guarantees that there exists a probability space $(\Omega, \mathcal{F}, P)$ supporting $W_{T}$ and $W_{1}$ such that $W_{T} \rightarrow \Lambda W_{1}$ a.s. uniformly in [0,1]. Moreover, we can state the following result, which follows from a strong approximation result due to Akonom (1993) (Theorem 3, p. 74).

Lemma 5.1 Under Assumption 2, we have that

a) $\sup _{r \in[0,1]}\left|W_{T}(r)-\Lambda W_{1}(r)\right|=O_{P}\left(T^{-1 / 2+1 / p}\right)$.

b) For any $\epsilon>0, P\left(\sup _{r \in[0,1]}\left|W_{T}(r)-\Lambda W_{1}(r)\right| \geq T^{-1 / 2+3 /(2 p)}\right)=o\left(T^{-\frac{1}{2}+\frac{3}{2 p}+\epsilon}\right)$.

Part a) of Lemma 5.1 shows that the stochastic order of $\sup _{r \in[0,1]}\left|W_{T}(r)-\Lambda W_{1}(r)\right|$ is equal to $O_{P}\left(T^{-1 / 2+1 / p}\right)$. As we will show next, the $t$-statistic can be written as a functional of $W_{T}(r)$ (or of its distributionally equivalent copy $T^{-1 / 2} \sum_{t=1}^{[T r]} u_{t}$ ). Thus, we can use part a) to determine the stochastic order of the error term in the stochastic expansion of the $t$-statistic. Part b) shows that $W_{T}$ can be approximated by $\Lambda W_{1}$ with an error that is distributionally ${ }^{5}$ of order $O\left(T^{-1 / 2+3 /(2 p)}\right)$. Thus, although the approximation error of $W_{T}$ with $\Lambda W_{1}$ is of order $O_{P}\left(T^{-1 / 2+1 / p}\right)$, its effect is distributionally of a larger order of magnitude, namely $O\left(T^{-1 / 2+3 /(2 p)}\right)$. We will rely on this result to derive the error of the fixed- $b$ asymptotic approximation. 


\subsection{Asymptotic expansion of the $t$-statistic}

We first provide an asymptotic expansion for the $t$-statistic. The $t$-statistic can be written as follows:

$$
t_{\hat{\beta}_{1}}=\frac{\sqrt{T}\left(\hat{\beta}_{1}-\beta_{1}\right)}{\sqrt{\hat{\Omega}}}
$$

where $\widehat{\beta}_{1}=\bar{y}$ and

$$
\hat{\Omega}=\hat{\Gamma}_{0}+2 \sum_{j=1}^{M}\left(1-\frac{j}{M}\right) \hat{\Gamma}_{j}, \text { with } \hat{\Gamma}_{j}=T^{-1} \sum_{t=j+1}^{T} \hat{u}_{t} \hat{u}_{t-j} .
$$

Thus, $\hat{\Omega}$ is the Bartlett kernel variance estimator of $\Omega=\lim _{T \rightarrow \infty} \operatorname{Var}\left(T^{-1 / 2} \sum_{t=1}^{T} u_{t}\right)=\sigma^{2} \pi^{2}(1)$. The bandwidth is equal to $M=b T$, where $b$ is a fixed constant. Following Kiefer and Vogelsang (2005), we can write

$$
\hat{\Omega}=2 b^{-1} T^{-2} \sum_{t=1}^{T-1} \hat{S}_{t}^{2}-2 b^{-1} T^{-2} \sum_{t=1}^{T-[b T]-1} \hat{S}_{t} \hat{S}_{t+[b T]},
$$

where $\hat{S}_{t}=\sum_{i=1}^{t} \hat{u}_{i}$ and $\hat{S}_{t}=S_{t}-\left(\frac{t}{T}\right) S_{T}$, with $S_{t}=\sum_{i=1}^{t} u_{i}$.

Lemma 5.2 Under Assumption 2, and for any fixed $b \in(0,1]$, we have

$$
\hat{\Omega}=\Omega Q_{1}(b)+O_{P}\left(T^{-1 / 2+1 / p}\right)
$$

with $Q_{1}(b)$ given by (4).

Lemma 5.2 provides an asymptotic expansion for $\hat{\Omega}$ with remainder $O_{P}\left(T^{-1 / 2+1 / p}\right)$. The leading term of this expansion is the distribution derived by Kiefer and Vogelsang (2005). The rate of convergence of $\hat{\Omega}$ increases with $p$, the number of finite moments of $\varepsilon$. If all moments of $\varepsilon$ exist, we can set $p=\infty$ and get the parametric convergence rate of $O_{P}\left(T^{-1 / 2}\right)$. Our next result provides the asymptotic expansion for the $t$-statistic.

Theorem 5.1 Under Assumption 2, and for any fixed $b \in(0,1]$, we have

$$
t_{\hat{\beta}_{1}}=\frac{W_{1}(1)}{\sqrt{Q_{1}(b)}}+O_{P}\left(T^{-1 / 2+1 / p}\right)
$$

where $t_{\hat{\beta}_{1}}$ and $Q_{1}(b)$ are defined as above.

The leading term of the expansion for $t_{\hat{\beta}_{1}}$ is the fixed- $b$ first-order asymptotic distribution derived by Kiefer and Vogelsang (2005). Using Lemma 5.1 b) and following Park (2003, Corollary 3.8) we can prove the following corollary to Theorem 5.1 . 
Corollary 5.1 Under Assumption 2, and for any fixed $b \in(0,1]$, we have

$$
P\left(t_{\hat{\beta}_{1}} \leq x\right)=P\left(\frac{W_{1}(1)}{\sqrt{Q_{1}(b)}} \leq x\right)+o\left(T^{-1 / 2+3 /(2 p)+\epsilon}\right),
$$

uniformly in $x \in \mathbb{R}$, for any $\epsilon>0$.

Corollary 5.1 gives an upper bound on the rate at which the true sampling distribution of $t_{\hat{\beta}_{1}}$ converges to the fixed- $b$ asymptotic approximation. When all moments exist (as in the Gaussian case considered in our simulations), $p=\infty$, then the bound on the error of the fixed- $b$ asymptotic approximation is of order $o\left(T^{-1 / 2+\epsilon}\right)$ for any $\epsilon>0$. For general linear GMM regression models, Sun and Phillips (2009) show that the error of the normal approximation for one-sided tests is of order $O\left(T^{-1 / 2}\right)+O\left(\frac{M}{T}\right)+O\left(M^{-1}\right)$ for the case of the Bartlett kernel (cf. their equation (41)). This result is derived from an Edgeworth expansion of the finite sample distribution of the test statistic and hence it provides a sharp bound on each of the three terms involved. The order of the error depends on the rate of the bandwidth. Suppose $M=c T^{\delta}$ for some constant $c$. When $\delta \in\left(0, \frac{1}{2}\right)$, the dominant term is the $O\left(M^{-1}\right)$ term, and it is larger than $O\left(T^{-1 / 2}\right)$. When $\delta \in\left(\frac{1}{2}, 1\right)$, the dominant term is the $O\left(\frac{M}{T}\right)$ term which is also larger than $O\left(T^{-1 / 2}\right)$. Only for $\delta=\frac{1}{2}$ do the three terms balance and equal $O\left(T^{-1 / 2}\right)$. Thus, any rate for $M$ that is either larger or smaller than $O(\sqrt{T})$ implies a normal approximation error of magnitude larger than $O\left(T^{-1 / 2}\right)$. This is unequivocally larger than our upper bound when $p=\infty$; when $p<\infty$, it can be larger or smaller than our upper bound depending on the particular value of $p$ and how it relates to the rate of $M$. For instance, $p>9$ suffices if $M=O\left(T^{1 / 3}\right)$, which is the rate of the conventional MSEoptimal bandwidth parameter choice for the Bartlett kernel. As Sun and Phillips (2009) show, the MSE-optimal bandwidth choice is suboptimal if the goal is to minimize the coverage probability error of a confidence interval. In this case, choosing $M=O(\sqrt{T})$ yields the smallest possible coverage error of order $O\left(T^{-1 / 2}\right)$ for the normal approximation. Because our upper bound is no smaller than $O\left(T^{-1 / 2}\right)$ and is $O\left(T^{-1 / 2}\right)$ only when $p=\infty$, our upper bound is uninformative when $M=O(\sqrt{T})$ and makes no prediction about the relative accuracy of the normal and fixed- $b$ approximations.

We should point out that stronger results than Corollary 5.1 have been obtained in some recent work if it assumed that $u_{t}$ is Gaussian. Jansson (2004) has established that (the bound on) the error of the fixed- $b$ asymptotic approximation is $O\left(\frac{\log T}{T}\right)$ for the case of the Bartlett kernel with $b=1$. This result has been refined to $O\left(T^{-1}\right)$ and extended to a general class of kernels and wider range of $b$ by Sun et al. (2008). In contrast, our bound is of order $O\left(T^{-1 / 2}\right)$ when $u_{t}$ is Gaussian, implying that the method of strong approximations does not deliver a sharp bound in the Gaussian case. While the error rate results of Jansson (2004) and Sun et al. (2008) are stronger than ours, it is not known whether they continue to hold without the Gaussian assumption. Because the 
Gaussian assumption cannot hold for the bootstrap, the methods of proof used by Jansson (2004) and Sun et al. (2008) cannot be directly applied to the bootstrap.

\subsection{Asymptotic expansion of the naive i.i.d. bootstrap $t$-statistic}

Next, we provide an asymptotic expansion for the naive i.i.d. bootstrap statistic. Let

$$
u_{t}^{*} \sim \text { i.i.d. }\left\{\hat{u}_{t}=y_{t}-\bar{y}: t=1, \cdots, T\right\}
$$

be an i.i.d. bootstrap sample. Note that $u_{t}^{*}=y_{t}^{*}-\bar{y}$, where $y_{t}^{*}$ is an i.i.d. bootstrap observation drawn from $\left\{y_{t}\right\}$. The naive i.i.d. bootstrap $t$-statistic is defined as

$$
t_{\hat{\beta}_{1}^{*}}=\frac{\sqrt{T}\left(\hat{\beta}_{1}^{*}-\hat{\beta}_{1}\right)}{\sqrt{\hat{\Omega}^{*}}}
$$

where $\hat{\beta}_{1}^{*}=\overline{y^{*}}$ and $\hat{\Omega}^{*}$ is of the same form as $\hat{\Omega}$ but evaluated with the bootstrap data:

$$
\hat{\Omega}^{*}=2 b^{-1} T^{-2} \sum_{t=1}^{T-1} \hat{S}_{t}^{* 2}-2 b^{-1} T^{-2} \sum_{t=1}^{T-[b T]-1} \hat{S}_{t}^{*} \hat{S}_{t+[b T]}^{*},
$$

where $\hat{S}_{t}^{*}=S_{t}^{*}-\left(\frac{t}{T}\right) S_{T}^{*}, \quad S_{t}^{*} \equiv \sum_{i=1}^{t} u_{i}^{*}$.

Let $\Omega_{T}^{*}=\operatorname{Var}^{*}\left(T^{-1 / 2} \sum_{t=1}^{T} u_{t}^{*}\right)$. We can show that $\Omega_{T}^{*}=T^{-1} \sum_{t=1}^{T} \operatorname{Var}^{*}\left(u_{t}^{*}\right)=T^{-1} \sum_{t=1}^{T} \hat{u}_{t}^{2}$, and

$$
\Omega^{*} \equiv p \lim \Omega_{T}^{*}=E\left(u_{t}^{2}\right)=\sigma^{2} \sum_{i=1}^{\infty} \pi_{i}^{2} \neq \sigma^{2} \pi^{2}(1)=\Omega,
$$

so the i.i.d. bootstrap does not consistently estimate the long run variance of $\hat{\beta}_{1}$. However, we will show that the i.i.d. bootstrap can still provide an asymptotic refinement over the $N(0,1)$ approximation.

By a bootstrap FCLT,

$$
W_{T}^{0 *}(r)=T^{-1 / 2} \sum_{t=1}^{[T r]} u_{t}^{*} \Rightarrow^{d_{*}} \Omega^{* 1 / 2} W_{1}(r),
$$

in probability, where $W_{1}$ denotes a standard Brownian motion independent of the realization of $u_{t}$. As above, we can find a process $W_{T}^{*}$ that has the same distribution as $W_{T}^{0 *}$, conditional on the original sample, and such that the following result follows. We write

$$
W_{T}^{*}(r)=T^{-1 / 2} \sum_{t=1}^{[T r]} u_{t}^{*}
$$

in probability, where the equality is to be interpreted as an equality in distribution under the bootstrap measure. The following result is a strong approximation for the bootstrap partial sum process. 
Lemma 5.3 Under Assumption 2, we have

a) $\sup _{r \in[0,1]}\left|W_{T}^{*}(r)-\Omega^{* 1 / 2} W_{1}(r)\right|=O_{P^{*}}\left(T^{-1 / 2+1 / p}\right)$, in probability.

b) For any $\epsilon>0, P^{*}\left(\sup _{r \in[0,1]}\left|W_{T}^{*}(r)-\Omega^{* 1 / 2} W_{1}(r)\right| \geq T^{-1 / 2+3 /(2 p)}\right)=o_{P}\left(T^{-1 / 2+3 /(2 p)+\epsilon}\right)$.

The next result gives an expansion for $\hat{\Omega}^{*}$ and is the bootstrap analogue of Lemma 5.2 .

Lemma 5.4 Under Assumption 2, we have

$$
\hat{\Omega}^{*}=\Omega^{*} Q_{1}(b)+O_{P^{*}}\left(T^{-1 / 2+1 / p}\right),
$$

in probability, where $Q_{1}(b)$ is as defined previously.

Given Lemma 5.4, we can derive the following asymptotic expansion for the naive i.i.d. bootstrap $t$-statistic.

Theorem 5.2 Under Assumption 2, we have

$$
t_{\hat{\beta}_{1}^{*}}=\frac{W_{1}(1)}{\left(Q_{1}(b)\right)^{1 / 2}}+O_{P^{*}}\left(T^{-1 / 2+1 / p}\right)
$$

in probability.

The following corollary to Theorem 5.2 shows that the effect of the remainder term in the asymptotic expansion of $t_{\hat{\beta}_{1}^{*}}$ is distributionally of order $O\left(T^{-1 / 2+3 /(2 p)}\right)$.

Corollary 5.2 Under Assumption 2, we have

$$
P^{*}\left(t_{\hat{\beta}_{1}^{*}} \leq x\right)=P\left(\frac{W(1)}{(Q(b))^{1 / 2}} \leq x\right)+o_{P}\left(T^{-1 / 2+3 /(2 p)+\epsilon}\right),
$$

uniformly in $x \in \mathbb{R}$, for any $\epsilon>0$.

It then follows from Corollaries 5.1 and 5.2 that

$$
\sup _{x \in \mathbb{R}}\left|P^{*}\left(t_{\hat{\beta}_{1}^{*}} \leq x\right)-P\left(t_{\hat{\beta}_{1}} \leq x\right)\right|=o_{P}\left(T^{-1 / 2+3 /(2 p)+\epsilon}\right),
$$

uniformly in $x \in \mathbb{R}$, for any $\epsilon>0$.

The result in (12) shows that the bound on the i.i.d. bootstrap error is of the same order of magnitude as the bound on the error implied by the fixed- $b$ asymptotic approximation. In particular, if $p=\infty$ the i.i.d. bootstrap error bound is arbitrarily close to $o_{P}\left(T^{-1 / 2+\epsilon}\right)$, smaller than the error implied by the normal approximation when $M=c T^{\delta}$, and $\delta \neq 1 / 2$. In particular, the i.i.d. bootstrap error is smaller than the error associated with the normal approximation when 
the optimal MSE bandwidth is used to compute the HAC Bartlett kernel estimator whenever $p>9$. When $M=O(\sqrt{T})$, the optimal-ERP bandwidth, the bound on the i.i.d. bootstrap error is of the same magnitude as the normal error when $p=\infty$ and it is larger when $p<\infty$. Therefore, no conclusions can be obtained in this case because our bound results are not sharp.

The reason why the i.i.d. bootstrap can provide a refinement in this context is that it replicates the fixed- $b$ distribution. This is true even when the data are dependent, as we showed more generally before.

\section{Heuristic Comparisons of Edgeworth and Fixed- $b$}

While rigorous comparisons of the Edgeworth approximations with fixed- $b$ approximations are well beyond the scope of this paper, some heuristic comparisons can be instructive for guiding future work. In deriving formal Edgeworth approximations, Velasco and Robinson (2001) approximate the bias and variance of the HAC variance estimator under the traditional assumption that $M / T$ shrinks to zero. In the simple location model we have from Velasco and Robinson (2001) for the QS kernel

$$
\begin{aligned}
\operatorname{bias}\left(\frac{\widehat{\Omega}}{\Omega}\right) & =E\left(\frac{\widehat{\Omega}}{\Omega}\right)-1 \approx-\frac{18}{125} \pi^{2} M^{-2} \Omega^{-1} \sum_{j=-\infty}^{\infty} j^{2} \Gamma_{j}-\frac{M}{T} \int_{0}^{1} k(s) d s \\
& =-\frac{18}{125} \pi^{2} M^{-2} \Omega^{-1} \sum_{j=-\infty}^{\infty} j^{2} \Gamma_{j}-\frac{M}{T} \frac{5}{4}, \\
\operatorname{Var}\left(\frac{\widehat{\Omega}}{\Omega}\right) & \approx\left(\frac{M}{T}\right) 2 \int_{0}^{1} k(s)^{2} d s=2 \frac{M}{T} .
\end{aligned}
$$

Although the Bartlett kernel does not satisfy the assumptions used by Velasco and Robinson (2001), existing results in the spectral analysis literature give for the Bartlett kernel

$$
\begin{aligned}
\operatorname{bias}\left(\frac{\widehat{\Omega}}{\Omega}\right) & \approx-M^{-1} \Omega^{-1} \sum_{j=-\infty}^{\infty} j \Gamma_{j}-\frac{M}{T} \int_{0}^{1} k(s) d s=-M^{-1} \Omega^{-1} \sum_{j=-\infty}^{\infty} j \Gamma_{j}-\frac{M}{T}, \\
\operatorname{Var}\left(\frac{\widehat{\Omega}}{\Omega}\right) & \approx\left(\frac{M}{T}\right) 2 \int_{0}^{1} k(s)^{2} d s=\frac{4}{3} \frac{M}{T} .
\end{aligned}
$$

Notice that the Edgeworth approximation (9) is a function of these moments.

Alternatively, the fixed- $b$ approximation approximates the entire distribution of $\widehat{\Omega}$ :

$$
\frac{\widehat{\Omega}}{\Omega} \approx Q_{1}(b) .
$$

The bias of $\widehat{\Omega} / \Omega$ can be approximated by $E\left(Q_{1}(b)-1\right)$. It is interesting to compare $E\left(Q_{1}(b)-1\right)$ and $\operatorname{Var}\left(Q_{1}(b)\right)$ with the traditional bias and variance formulas. For the Bartlett kernel (see Kiefer 
and Vogelsang (2005))

$$
\begin{aligned}
E\left(Q_{1}(b)-1\right) & =-b+\frac{1}{3} b^{2} \\
\operatorname{Var}\left(Q_{1}(b)\right) & =\frac{4}{3} b-\frac{7}{3} b^{2}+\frac{14}{15} b^{3}+\frac{2}{9} b^{4} \text { for } b \leq \frac{1}{2} .
\end{aligned}
$$

Recalling that $b=M / T$ we see that the moments of the fixed- $b$ asymptotic distribution match the traditional moments for terms of order $M / T$. The differences between the two approximations are that the fixed- $b$ approximation does not include the $-M^{-1} \Omega^{-1} \sum_{j=-\infty}^{\infty} j \Gamma_{j}$ bias term because it is $o(1)$ under fixed- $b$ asymptotics, but the fixed- $b$ approximation includes terms of order $(M / T)^{2}$ and higher.

These heuristic observations can shed some light on some of the patterns observed in Figures 1-4. In the case of i.i.d. errors, the bias term of order $M^{-1}$ is exactly zero (because $\left.\sum_{j=-\infty}^{\infty}|j| \Gamma_{j}=\sum_{j=-\infty}^{\infty} j^{2} \Gamma_{j}=0\right)$ and the main difference between the fixed- $b$ approximation and the Edgeworth approximation are the higher order terms in the bias and variance formulas for the fixed- $b$ approximation. When $M$ is small, the difference between the Edgeworth and fixed- $b$ approximations are negligible whereas for large $M$, the fixed- $b$ approximation is slightly more accurate. When $\rho=0.3$, the Edgeworth approximation is more accurate when $M$ is small because it picks up the $M^{-1}$ term whereas for larger $M$, the fixed- $b$ approximation is more accurate. These differences become more apparent when $\rho=0.9$.

An intriguing possibility is apparent. Because of the asymptotic equivalence between fixed- $b$ asymptotics and the naive i.i.d. bootstrap, it appears the i.i.d. bootstrap captures the influence of the bias and variance of $\widehat{\Omega}$ to higher orders than the Edgeworth approximation with respect to terms that depend on powers of $M / T$, but the i.i.d. bootstrap does not capture the bias term that depends on $\sum_{j=-\infty}^{\infty}|j| \Gamma_{j}$ or $\sum_{j=-\infty}^{\infty} j^{2} \Gamma_{j}$. With careful choice of block length, the naive block bootstrap could capture these bias term while continuing to capture the $M / T$ and higher order terms terms. The simulations reported in Figures 1-4 show that when there is serial correlation in the data, increasing the block length from 1 to 5 does improve the approximation. It is possible this improvement is coming at least in part through the first bias term.

\section{Conclusion}

In this paper, we theoretically analyze the performance of the naive MBB applied to HAC robust tests based on nonparametric kernel estimators of the long run variance. In simulations reported here and in Kiefer and Vogelsang (2005) it was found that the naive MBB outperforms the $N(0,1)$ approximation in finite samples. This improvement holds for many kernels, including the Bartlett 
kernel, and holds even for the i.i.d. bootstrap, despite the dependence in the data. These simulations suggest that the performance of the naive $\mathrm{MBB}$ is tightly linked to the finite sample performance of the recently developed fixed- $b$ (i.e. fixed bandwidth) asymptotics. We provide a theoretical explanation for this result: we prove that the bootstrap distribution of the naive MBB is asymptotically the same as the fixed- $b$ asymptotic distribution. In addition, for a simple location model we show that a naive i.i.d. bootstrap can reduce the magnitude of the error in estimating one-sided distribution functions of robust $t$ - statistics compared to the $N(0,1)$ approximation error for statistics studentized with a Bartlett kernel variance estimator depending on the bandwidth choice and the number of finite moments in the data. Our simulations also suggest that the naive MBB can be more accurate than the fixed- $b$ asymptotic approximation when the block size is chosen appropriately. Providing a theoretical explanation for this finding is a challenging topic of for future research. 


\section{Notes}

${ }^{1}$ The finding that the naive bootstrap is first order equivalent to the fixed- $b$ asymptotic distribution for fixed block lengths in addition to slowly growing block lengths does not suggest that the choice of block length does not matter. Our simulations will clearly show that the choice of block length matters and the simulations suggest the naive bootstrap can provide refinements over fixed- $b$ asymptotics with suitable choice of block length. We conjecture that such refinements will only hold for block lengths that grow at a suitable rate with fixed block lengths being insufficient. It should also be pointed out that from the perspective of improvements over the standard normal approximation, our results do depart from the existing literature by showing that use of small/fixed block lengths work for the naive bootstrap. This is a positive result because it shows that a wide range of block length choices can deliver improvements over the standard normal approximation when the naive bootstrap is used. This is a desirable robustness.

${ }^{2}$ The regularity conditions used by Velasco and Robinson (2001) appear to exclude the Bartlett and Parzen kernels because their spectral windows do not truncate outside the range $[-\pi, \pi]$ although our simulation results suggest that their results likely hold for the Bartlett kernel. Velasco and Robinson (2001) conjecture that their proofs could be modified to allow kernels like the Bartlett and Parzen.

${ }^{3}$ In unreported simulations we obtained results for the case of $\rho=0.99$. As one would expect, the tendency to over-reject increases even for the NB5 and GK5 bootstraps.

${ }^{4}$ We do not consider size-adjusted power because there is only one statistic being considered and its size adjusted power has nothing to do with the choice of critical value. Only unadjusted power depends on the choice of critical value.

${ }^{5}$ We follow Park (2003) and say that a random sequence $R_{T}$ is distributionally of order $o\left(T^{-a+\epsilon}\right)=$ $O\left(T^{-a}\right)$ if $P\left(\left|R_{T}\right|>T^{-a}\right)=O\left(T^{-a}\right)$ for some $\epsilon>0$.

\section{References}

Akonom, J.: (1993), Comportement asymptotique du temps d'occupation du processus des sommes partielles, Annales de l'Institut Henri Poincare 29, 57-81.

Andrews, D. W. K.: (1991), Heteroskedasticity and autocorrelation consistent covariance matrix estimation, Econometrica 59, 817-854.

Andrews, D. W. K.: (2002), Higher-order improvements of a computationally attactive k-step bootstrap for extremum estimators, Econometrica 70, 119-162.

Billingsley, P.: (1968), Convergence of Probability Measures, Wiley, New York. 
Chang, Y. and Park, J.: (2003), A sieve bootstrap for the test of a unit root, Journal of Time Series Analysis 24, 379-400.

Davidson, J.: (1994), Stochastic Limit Theory, Oxford University Press, New York.

Davison, A. C. and Hall, P.: (1993), On studentizing and blocking methods for implementing the bootstrap with dependent data, Australian Journal of Statistics 35, 215-224.

de Jong, R. M. and Davidson, J.: (2000), Consistency of kernel estimators of heteroskedastic and autocorrelated covariance matrices, Econometrica 68, 407-424.

Fitzenberger, B.: (1997), The moving blocks bootstrap and robust inference for linear least squares and quantile regressions, Journal of Econometrics 82, 235-287.

Götze, F. and Künsch, H. R.: (1996), Second-order correctness of the blockwise bootstrap for stationary observations, Annals of Statistics 24, 1914-1933.

Hall, P. and Horowitz, J. L.: (1996), Bootstrap critical values for tests based on generalized method of moments estimators, Econometrica 64, 891-916.

Hansen, B. E.: (1991), Strong laws for dependent heterogeneous processes, Econometric Theory 7, 213-221.

Hansen, B. E.: (1992), Erratum, Econometric Theory 8, 421-422.

Inoue, A. and Shintani, M.: (2006), Bootstrapping gmm estimators for time series, Journal of Econometrics 127(2), 531-555.

Jansson, M.: (2004), The error rejection probability of simple autocorrelation robust tests, Econometrica 72, 937-946.

Kiefer, N. M. and Vogelsang, T. J.: (2005), A new asymptotic theory for heteroskedasticityautocorrelation robust tests, Econometric Theory 21, 1130-1164.

Kunsch, H. R.: (1989), The jackknife and the bootstrap for general stationary observations, Annals of Statistics 17, 1217-1241.

Lahiri, S. N.: (1996), On edgeworth expansion and moving block bootstrap for studentized mestimators in multiple linear regression models, Journal of Multivariate Analysis 56, 42-59.

Liu, R. and Singh, K.: (1992), Moving blocks jackknife and bootstrap capture weak dependence, in R. LePage and L. Billiard (eds), Exploring the Limits of the Bootstrap, Wiley, New York. 
Paparoditis, E. and Politis, P.: (2003), Residual-based block bootstrap for unit root testing, Econometrica 71, 813-855.

Park, J. Y.: (2003), Bootstrap unit root tests, Econometrica 71, 1845-1898.

Park, J. Y.: (2006), A bootstrap theory for weakly integrated processes, Journal of Econometrics 133, 639-672.

Phillips, P. C. B. and Solo, V.: (1992), Asymptotics for linear processes, The Annals of Statistics 20, 971-1001.

Sakhanenko, A. I.: (1980), On unimprovable estimates of the rate of convergence in invariance principle, Colloquia Mathematica Societatis Janos Bolyai 32, Nonparametric Statistical Inference, Budapest, Hungary, pp. 779-783.

Singh, K.: (1981), On the asymptotic accuracy of efron's bootstrap, Annals of Statistics 9, 11871195 .

Sun, Y. and Phillips, P.: (2009), Optimal Bandwidth Choice for Interval Estimation in GMM Regression. Working Paper, Department of Economics, UCSD.

Sun, Y., Phillips, P. C. B. and Jin, S.: (2008), Optimal bandwidth selection in heteroskedasticityautocorrelation robust testing, Econometrica 76, 175-194.

Velasco, C. and Robinson, P. M.: (2001), Edgeworth expansions for spectral density estimates and studentized sample mean, Econometric Theory 17, 497-539.

\section{Appendix A}

This Appendix contains the proofs of the results in Section 4. Throughout this Appendix $K$ denotes a generic constant that may change from one usage to the next. We first state four lemmas that are auxiliary in proving Lemma 4.1 and Theorem 4.1 in Section 4. We then provide the proofs of our main results followed by the proofs of the auxiliary lemmas.

The following result is a maximal inequality for mixingales (see e.g. Davidson (1994) for a definition of mixingale) due to Hansen (1991, 1992). Zero mean NED processes on a mixing process are mixingales and we will repeatedly use this result in our proofs.

Lemma A.1 For some nondecreasing sequence of $\sigma$-fields $\left\{\mathcal{F}^{t}\right\}$ and for some $p>1$, let $\left\{X_{t}, \mathcal{F}^{t}\right\}$ be an $L_{p}$-mixingale with mixingale coefficients $\psi_{m}$ and mixingale constants $c_{t}$. Then, letting $S_{j}=$ $\sum_{t=1}^{j} X_{t}$ and $\Psi=\sum_{m=1}^{\infty} \psi_{m}$ it follows that

a) If $1<p \leq 2,\left\|\max _{j \leq T}\left|S_{j}\right|\right\|_{p} \leq K \Psi\left(\sum_{t=1}^{T} c_{t}^{p}\right)^{1 / p}$. 
b) For $p \geq 2,\left\|\max _{j \leq T}\left|S_{j}\right|\right\|_{p} \leq K \Psi\left(\sum_{t=1}^{T} c_{t}^{2}\right)^{1 / 2}$.

The following result gives the probability limits of the MBB variance of a scaled bootstrap sample mean under two different assumptions on the block size $\ell$ : (a) when $\ell$ is fixed as $T \rightarrow \infty$; and (b) when $\ell \rightarrow \infty$ as $T \rightarrow \infty$ at an appropriate rate. We state the result for a general time series $\left\{X_{t}\right\}$ satisfying the following assumptions:

Assumption A Let $\left\{X_{t}\right\}$ be a weakly stationary sequence of $s \times 1$ random vectors such that the following hold:

(i) For some $p>2,\left\|X_{t}\right\|_{p} \leq \Delta<\infty$ for all $t=1,2, \ldots$.

(ii) $\left\{X_{t}\right\}$ is $L_{2}$-NED on $\left\{V_{t}\right\}$ with NED coefficients of size $-1 / 2$.

(iii) $\left\{V_{t}\right\}$ is $\alpha$-mixing of size $-\frac{p}{p-2}$.

Let $\left\{X_{t}^{*}: t=1,2, \ldots, T\right\}$ denote a MBB resample obtained from $\left\{X_{t}: t=1,2, \ldots, T\right\}$ using block size $\ell$. Let $\Omega_{T}^{*}=\operatorname{Var}^{*}\left(T^{-1 / 2} \sum_{t=1}^{T} X_{t}^{*}\right)$ denote the bootstrap variance of $\sqrt{T} \bar{X}_{T}^{*}$.

Lemma A.2 Suppose $\left\{X_{t}\right\}$ satisfies Assumption A. Then,

a) For any fixed $\ell$ such that $1 \leq \ell<T$, as $T \rightarrow \infty$,

$$
p \lim _{T \rightarrow \infty} \Omega_{T}^{*}=\Gamma_{0}+\sum_{j=1}^{\ell}\left(1-\frac{j}{\ell}\right)\left(\Gamma_{j}+\Gamma_{j}^{\prime}\right) \equiv \Omega_{\ell},
$$

where $\Gamma_{j}=E\left(\left(X_{t}-\mu\right)\left(X_{t-j}-\mu\right)^{\prime}\right), \mu=E\left(X_{t}\right)$.

b) Let $\ell=\ell_{T} \rightarrow \infty$ as $T \rightarrow \infty$ such that $\ell^{2} / T \rightarrow 0$. Then

$$
p \lim _{T \rightarrow \infty} \Omega_{T}^{*}=\Gamma_{0}+\sum_{j=1}^{\infty}\left(\Gamma_{j}+\Gamma_{j}^{\prime}\right) \equiv \Omega .
$$

Our next result establishes a FCLT for the bootstrap partial sum process $Z_{T}^{*}(r)=T^{-1 / 2} \sum_{t=1}^{[r T]}\left(X_{t}^{*}-E^{*}\left(X_{t}^{*}\right)\right)$. We need to strengthen Assumption A as follows.

Assumption $\mathbf{A}^{\prime}$ Let $\left\{X_{t}\right\}$ be a weakly stationary sequence of $s \times 1$ random vectors such that the following hold:

a) For some $p>2$ and some $\delta>0,\left\|X_{t}\right\|_{p+\delta} \leq \Delta<\infty$ for all $t=1,2, \ldots$

b) $\left\{X_{t}\right\}$ is $L_{2+\delta}$-NED on $\left\{V_{t}\right\}$ of size -1 .

c) $\left\{V_{t}\right\}$ is $\alpha$-mixing of size $-\frac{(2+\delta)(p+\delta)}{p-2}$. 
Lemma A.3 Suppose Assumption $A^{\prime}$ holds and let $\Omega_{\ell}$ and $\Omega$ as defined in Lemma A.2 be positive definite matrices. It follows that

a) For any fixed $\ell$ such that $1 \leq \ell<T$, as $T \rightarrow \infty$,

$$
Z_{T}^{*}(r) \Rightarrow^{P^{*}} \Lambda_{\ell} W_{s}(r)
$$

in probability, where $\Lambda_{\ell}$ is the square root matrix of $\Omega_{\ell}$.

b) Let $\ell=\ell_{T} \rightarrow \infty$ as $T \rightarrow \infty$ such that $\ell^{2} / T \rightarrow 0$. Then

$$
Z_{T}^{*}(r) \Rightarrow^{P^{*}} \Lambda W_{s}(r)
$$

in probability, where $\Lambda$ is the square root matrix of $\Omega$.

The following result will be used in the proof of Lemma 4.1.

Lemma A.4 Suppose $\left\{X_{t}-E\left(X_{t}\right)\right\}$ is a weakly stationary $L_{2}$-mixingale with $\left\|X_{t}\right\|_{p} \leq \Delta<\infty$ for some $p>2$ such that its mixingale coefficients $\psi_{m}$ satisfy $\sum_{m=1}^{\infty} \psi_{m}<\infty$ and its mixingale constants are uniformly bounded. Let $\left\{X_{t}^{*}: t=1, \ldots, T\right\}$ denote a MBB resample of $\left\{X_{t}: t=1, \ldots, T\right\}$ with block size $\ell$ satisfying either of the two following conditions: a) $\ell$ is fixed as $T \rightarrow \infty$, or b) $\ell \rightarrow \infty$ as $T \rightarrow \infty$ with $\ell=o(T)$. Then, for any $\eta>0$, as $T \rightarrow \infty$,

$$
P^{*}\left(\sup _{0 \leq r \leq 1}\left|T^{-1} \sum_{t=1}^{[r T]}\left(X_{t}^{*}-E^{*}\left(X_{t}^{*}\right)\right)\right|>\eta\right)=o_{P}(1) \text {. }
$$

Proof of Lemma 4.1. We start with the proof of a) and b), which can be treated simultaneously. Given our definitions of $v_{0 t}^{*}$ and $v_{t}^{*}$, we can write $v_{t}^{*}=v_{0 t}^{*}-x_{t}^{*} x_{t}^{* \prime}(\hat{\beta}-\beta)$, which implies that

$$
\begin{aligned}
X_{T}^{*}(r) & =T^{-1 / 2} \sum_{t=1}^{[r T]}\left(v_{0 t}^{*}-E^{*} v_{0 t}^{*}\right)+T^{-1 / 2} \sum_{t=1}^{[r T]} E^{*}\left(v_{0 t}^{*}\right)-T^{-1 / 2} \sum_{t=1}^{[r T]} x_{t}^{*} x_{t}^{* \prime}(\hat{\beta}-\beta) \\
& \equiv Z_{T}^{*}(r)+A_{1 T}^{*}(r)-A_{2 T}^{*}(r) .
\end{aligned}
$$

An application of Lemma A.3 implies that under Assumption $1^{\prime}, Z_{T}^{*}(r) \Rightarrow^{P^{*}} \Lambda^{*} W_{s}(r)$, in probability, where $\Lambda^{*}$ is the square root matrix of $\Omega^{*}=p \lim \operatorname{Var}^{*}\left(T^{-1 / 2} \sum_{t=1}^{T} v_{0 t}^{*}\right)$. In particular, by Lemma A.2, $\Omega^{*}=\Omega_{\ell}$ in a) and $\Omega^{*}=\Omega$ in b). Thus, to prove that $X_{T}^{*}(r) \Rightarrow{ }^{*} \Lambda^{*} W_{s}(r)$, in probability, it suffices to show that $\sup _{r}\left|A_{1 T}^{*}(r)-A_{2 T}^{*}(r)\right|=o_{P^{*}}(1)$ in probability. Adding and subtracting $T^{-1 / 2} \sum_{t=1}^{[r T]} E^{*}\left(x_{t}^{*} x_{t}^{* \prime}\right)(\hat{\beta}-\beta)$, and rearranging terms yields

$$
\begin{aligned}
A_{1 T}^{*}(r)-A_{2 T}^{*}(r) & =T^{-1 / 2} \sum_{t=1}^{[r T]} E^{*}\left(x_{t}^{*}\left(y_{t}^{*}-x_{t}^{* \prime} \hat{\beta}\right)\right)-T^{-1 / 2} \sum_{t=1}^{[r T]}\left(x_{t}^{*} x_{t}^{* \prime}-E^{*} x_{t}^{*} x_{t}^{* \prime}\right)(\hat{\beta}-\beta) \\
& \equiv B_{1 T}^{*}(r)-B_{2 T}^{*}(r) .
\end{aligned}
$$


Next, we show that $\sup _{r \in[0,1]}\left|B_{1 T}^{*}(r)\right|=o_{P^{*}}(1)$ and $\sup _{r \in[0,1]}\left|B_{2 T}^{*}(r)\right|=o_{P^{*}}(1)$, in probability. Note that

$$
\begin{aligned}
B_{1 T}^{*}(r) & =E^{*}\left(T^{-1 / 2} \sum_{m=1}^{M_{r}} \sum_{s=1}^{B} \hat{v}_{I_{m}+s}\right)=E^{*}\left(T^{-1 / 2} \sum_{m=1}^{M_{r}} \sum_{s=1}^{\ell} \hat{v}_{I_{m}+s}\right)-T^{-1 / 2} E^{*}\left(\sum_{s=B+1}^{\ell} \hat{v}_{I_{M_{r}}+s}\right) \\
& \equiv b_{1 T}^{*}(r)-b_{2 T}^{*}(r),
\end{aligned}
$$

where $M_{r}, B$ and $I_{m}$ are as defined in the proof of LemmaA.3. We can show that $\sup _{r \in[0,1]}\left|b_{2 T}^{*}(r)\right|=$ $O_{P^{*}}\left(k_{0}^{-1 / 2}\right)$ in probability, given in particular the fact $\hat{\beta}-\beta=O_{P}\left(T^{-1 / 2}\right)$. Moreover,

$$
b_{1 T}^{*}(r)=M_{r} T^{-1 / 2} E^{*}\left(\sum_{s=1}^{\ell} \hat{v}_{I_{1}+s}\right)=M_{r} \frac{\ell}{T^{1 / 2}} E^{*}\left(\hat{U}_{1}^{*}\right),
$$

where $\hat{U}_{1}^{*}=\ell^{-1} \sum_{s=1}^{\ell} \hat{v}_{I_{1}+s}$. Defining $\bar{X}_{T} \equiv T^{-1} \sum_{t=1}^{T} X_{t}$ for any random variables $X_{t}$, it is well known that $E^{*}\left({\overline{\hat{v}^{*}}}_{T}\right)=E^{*}\left(\hat{U}_{1}^{*}\right)=\overline{\hat{v}}_{T}+O_{P}\left(\frac{\ell}{T}\right)$ (see e.g. Fitzenberger (1997)). Since $\overline{\hat{v}_{T}}=0$ by the FOC defining the OLS estimator, it follows that $E^{*}\left(\hat{U}_{1}^{*}\right)=0+O_{P}\left(\frac{\ell}{T}\right)$, and noting that $\sup _{r \in[0,1]}\left|M_{r}\right|=k_{0}$, we have

$$
\sup _{r \in[0,1]}\left|b_{1 T}^{*}(r)\right|=\sup _{r \in[0,1]}\left|M_{r}\right| \frac{\ell}{T^{1 / 2}} E^{*}\left(\hat{U}_{1}^{*}\right)=k_{0} \frac{\ell}{T^{1 / 2}}\left(0+O_{P}\left(\frac{\ell}{T}\right)\right)=O_{P}\left(\frac{\ell}{\sqrt{T}}\right)=o_{P}(1),
$$

under both conditions a) and b). For $B_{2 T}^{*}(r)$, note that

$$
\sup _{r \in[0,1]}\left|B_{2 T}^{*}(r)\right|=\sup _{r \in[0,1]}\left|T^{-1} \sum_{t=1}^{[r T]}\left(x_{t}^{*} x_{t}^{* \prime}-E^{*} x_{t}^{*} x_{t}^{* \prime}\right)\right||\sqrt{T}(\hat{\beta}-\beta)| .
$$

Since $|\sqrt{T}(\hat{\beta}-\beta)|=O_{P}(1)$, it suffices to show that $\sup _{r \in[0,1]}\left|T^{-1} \sum_{t=1}^{[r T]}\left(x_{t}^{*} x_{t}^{* \prime}-E^{*} x_{t}^{*} x_{t}^{* \prime}\right)\right|=$ $o_{P^{*}}(1)$ in probability. This follows by an application of Lemma A.4 since $z_{t}=x_{t} x_{t}^{\prime}-E\left(x_{t} x_{t}^{\prime}\right)$ satisfies the assumptions of this lemma under Assumption 1. To prove c), note we can write

$$
\sup _{r \in[0,1]}\left|T^{-1} \sum_{t=1}^{[r T]} x_{t}^{*} x_{t}^{* \prime}-r Q\right| \leq I_{1}+I_{2}+I_{3}
$$

where $I_{1} \equiv \sup _{r \in[0,1]}\left|T^{-1} \sum_{t=1}^{[r T]}\left(x_{t}^{*} x_{t}^{* \prime}-E^{*}\left(x_{t}^{*} x_{t}^{* \prime}\right)\right)\right|, I_{2} \equiv \sup _{r \in[0,1]}\left|T^{-1} \sum_{t=1}^{[r T]}\left(E^{*}\left(x_{t}^{*} x_{t}^{* \prime}\right)-x_{t} x_{t}^{\prime}\right)\right|$, and $I_{3} \equiv \sup _{r \in[0,1]}\left|T^{-1} \sum_{t=1}^{[r T]} x_{t} x_{t}^{\prime}-r Q\right|$. As just proven, $I_{1}=o_{P^{*}}(1)$, in probability, and $I_{3}=$ $o_{P}(1)$ given Assumption 1. Next, we will show that $I_{2}=o_{P}(1)$. Adding and subtracting $\mu \equiv E\left(x_{t} x_{t}^{\prime}\right)$ yields

$$
I_{2} \leq T^{-1} \sup _{r \in[0,1]}\left|\sum_{t=1}^{[r T]} E^{*}\left(x_{t}^{*} x_{t}^{* \prime}-\mu\right)\right|+T^{-1} \sup _{r \in[0,1]}\left|\sum_{t=1}^{[r T]}\left(x_{t} x_{t}^{\prime}-\mu\right)\right| \equiv i_{1}+i_{2} .
$$

Under Assumption 1.a)-b) and 1.e), we can show that $\left\{x_{t} x_{t}^{\prime}-\mu\right\}$ is an $L_{2}$-NED of size -1 on $\left\{\varepsilon_{t}\right\}$ (cf. 
Davidson, 1994, Example 17.17). It then follows by Davidson's (1994) Theorem 17.5 that $\left\{x_{t} x_{t}^{\prime}-\mu\right\}$ is an $L_{2}$-mixingale of size -1 with uniformly bounded mixingale constants. Thus, $i_{2}=O_{P}\left(T^{-1 / 2}\right)$ by an application of Lemma A.1. Similarly, we can show that $i_{1}=O_{P}\left(T^{-1 / 2}\right)+O_{P}\left(\frac{\ell}{T}\right)$, which is $o_{P}(1)$ under our assumptions. Indeed, we can write

$$
i_{1}=k_{0}^{-1} \sup _{r \in[0,1]}\left|\sum_{m=1}^{M_{r}} E^{*}\left(U_{m}^{*}\right)\right|+O_{P}\left(\frac{\ell^{1 / 2}}{T}\right),
$$

where $U_{m}^{*}=\ell^{-1} \sum_{s=1}^{\ell}\left(x_{I_{m}+s} x_{I_{m}+s}^{\prime}-\mu\right)$. It follows that

$$
k_{0}^{-1} \sup _{r \in[0,1]}\left|\sum_{m=1}^{M_{r}} E^{*}\left(U_{m}^{*}\right)\right| \leq k_{0}^{-1} k_{0}\left|E^{*}\left(U_{1}^{*}\right)\right|=\left|T^{-1} \sum_{t=1}^{T}\left(x_{t} x_{t}^{\prime}-\mu\right)\right|+O_{P}\left(\frac{\ell}{T}\right),
$$

because

$$
E^{*}\left(U_{1}^{*}\right)=E^{*}\left(T^{-1} \sum_{t=1}^{T}\left(x_{t}^{*} x_{t}^{*}-\mu\right)\right)=T^{-1} \sum_{t=1}^{T}\left(x_{t} x_{t}^{\prime}-\mu\right)+O_{P}\left(\frac{\ell}{T}\right) .
$$

This completes the proof because $T^{-1} \sum_{t=1}^{T}\left(x_{t} x_{t}^{\prime}-\mu\right)=O_{P}\left(T^{-1 / 2}\right)$.

Proof of Theorem 4.1. The proof follows from Lemma 4.1, using the same arguments as in Kiefer and Vogelsang (2005).

Proof of Lemma A.2. As is well known, the MBB variance $\Omega_{T}^{*}$ is equal to the Bartlett kernel variance estimator of $\sqrt{T} \bar{X}_{T}$, up to a term of order $O_{P}\left(\frac{\ell^{2}}{T}\right)$ (see e.g. Fitzenberger $(1997$, p. 252)). Note that this term vanishes in probability under both sets of conditions on $\ell$. Result b) follows by de Jong and Davidson (2000) Theorem 2.1. Result a) follows by an argument similar to Liu and Singh (1992) and Fitzenberger (1997), under our more general dependence Assumption A.

Proof of Lemma A.3. These results are multivariate versions of a univariate FCLT given in Paparoditis and Politis (2003) (henceforth PP (2003)). Whereas PP (2003) assume a mixing condition on $\left\{X_{t}\right\}$, here we allow for the more general NED condition. Note that we assume throughout that $E\left(X_{t}\right)=0$ for all $t$ without loss of generality given that $\left\{X_{t}\right\}$ is stationary. Since Assumption A' implies Assumption A, it follows by Lemma A.2 that $p \lim _{T \rightarrow \infty} \Omega_{T}^{*}=\Omega^{*}$, where $\Omega^{*}$ is equal to $\Omega_{\ell}$ in a) and equal to $\Omega$ in b). Since by assumption both $\Omega_{\ell}$ and $\Omega$ are positive definite, $\Omega_{T}^{*-1 / 2}$ exists in probability for all $T$ sufficiently large. By the functional Cramer-Wold device, it suffices to show that $\lambda^{\prime} \Omega_{T}^{*-1 / 2} Z_{T}^{*}(r) \Rightarrow^{P^{*}} \lambda^{\prime} W_{s}(r)$ in probability for any $\lambda$ such that $\lambda^{\prime} \lambda=1$. Following PP (2003), for any $r \in[0,1]$, we can write

$$
W_{T}^{*}(r) \equiv \lambda^{\prime} \Omega_{T}^{*-1 / 2} Z_{T}^{*}(r)=\lambda^{\prime} \Omega_{T}^{*-1 / 2} T^{-1 / 2} \sum_{m=1}^{M_{r}} \sum_{s=1}^{B}\left(X_{I_{m}+s}-E^{*}\left(X_{I_{m+s}}\right)\right),
$$

where $M_{r}=[([r T]-1) / \ell]+1$ and $B=\min \{\ell,[r T]-(m-1) \ell\}$. Here, $I_{1}, \ldots, I_{k_{0}}$ are i.i.d. uniformly distributed on $\{0,1, \ldots, T-\ell\}$. Notice that for $r \in[0,1], M_{r} \in\left\{1, \ldots, k_{0}\right\}$ and 
$B \in\{1, \ldots, \ell\}$. As in PP (2003), we can write

$$
\begin{gathered}
W_{T}^{*}(r)=\lambda^{\prime} \Omega_{T}^{*-1 / 2} T^{-1 / 2} \sum_{m=1}^{M_{r}} \sum_{s=1}^{\ell}\left(X_{I_{m}+s}-E^{*}\left(X_{I_{m+s}}\right)\right) \\
-\lambda^{\prime} \Omega_{T}^{*-1 / 2} T^{-1 / 2} \sum_{s=B+1}^{\ell}\left(X_{I_{M_{r}+s}}-E^{*}\left(X_{I_{M_{r}+s}}\right)\right) \equiv W_{1 T}^{*}(r)-W_{2 T}^{*}(r) .
\end{gathered}
$$

The proof consists of two steps: (1) Show that $\sup _{r \in[0,1]}\left|W_{2 T}^{*}(r)\right|=O_{P^{*}}\left(k_{0}^{-1 / 2}\right)$ in probability; and (2) Show that $W_{1 T}^{*}(r) \Rightarrow^{P^{*}} W_{1}(r)$ in probability.

We start with (1). Since $\Omega_{T}^{*} \rightarrow \Omega^{*}$ in probability, and $\Omega^{*}$ is p.d., it follows that $\Omega_{T}^{*-1 / 2}=O_{P}(1)$. Thus, it suffices to show that

$$
E^{*}\left(\sup _{r \in[0,1]}\left|T^{-1 / 2} \sum_{s=B+1}^{\ell}\left(X_{I_{M_{r}}+s}-E^{*} X_{I_{M_{r}+s}}\right)\right|\right)=O_{P}\left(k_{0}^{-1 / 2}\right),
$$

by Markov's inequality. Since $k_{0}=T / \ell, k_{0} \rightarrow \infty$ as $T \rightarrow \infty$ under both set of conditions on $\ell$, which implies that $\sup _{r}\left|W_{2 T}^{*}(r)\right|=o_{P^{*}}(1)$ in probability. An application of triangle's inequality and Jensen's inequality implies that

$$
E^{*}\left(\sup _{r \in[0,1]}\left|T^{-1 / 2} \sum_{s=B+1}^{\ell}\left(X_{I_{M_{r}}+s}-E^{*} X_{I_{M_{r}+s}}\right)\right|\right) \leq 2 E^{*}\left(\sup _{r \in[0,1]}\left|T^{-1 / 2} \sum_{s=B+1}^{\ell} X_{I_{M_{r}}+s}\right|\right) .
$$

Since $I_{M_{r}} \sim$ i.i.d. Uniform $\{0, \ldots, T-\ell\}$, we have that

$$
E^{*}\left(\sup _{r \in[0,1]}\left|T^{-1 / 2} \sum_{s=B+1}^{\ell} X_{I_{M_{r}}+s}\right|\right)=\frac{1}{T-\ell+1} \sum_{j=0}^{T-\ell} \sup _{r \in[0,1]}\left|T^{-1 / 2} \sum_{s=B+1}^{\ell} X_{j+s}\right| .
$$

By Markov's inequality, (15) follows from $E\left(k_{0}^{1 / 2} E^{*}\left(\sup _{r \in[0,1]}\left|T^{-1 / 2} \sum_{s=B+1}^{\ell} X_{I_{M_{r}}+s}\right|\right)\right)=O(1)$. Recall that for $r \in[0,1], B \in\{1, \ldots, \ell\}$. Thus,

$k_{0}^{1 / 2} E\left(\sup _{r \in[0,1]}\left|T^{-1 / 2} \sum_{s=B+1}^{\ell} X_{j+s}\right|\right) \leq k_{0}^{1 / 2} T^{-1 / 2} E\left(\max _{1 \leq i \leq \ell}\left|\sum_{s=j+i}^{j+\ell} X_{s}\right|\right) \leq k_{0}^{1 / 2} T^{-1 / 2}\left\|\max _{1 \leq i \leq \ell}\left|\sum_{s=j+i}^{j+\ell} X_{s}\right|\right\| \|_{2}$.

We now apply Lemma A.1. Under Assumption $\mathrm{A}^{\prime},\left\{X_{t}\right\}$ is an $L_{2+\delta^{-}}$-mixingale (hence an $L_{2^{-}}$ mixingale) with mixingale coefficients $\psi_{m}$ of size -1 , hence $\Psi=\sum_{m=1}^{\infty} \psi_{m}<\infty$. In particular, we apply Theorem 17.5 of Davidson (1994), with $r=p+\delta, p=2+\delta, b=1$ and $a=\frac{(2+\delta)(p+\delta)}{p-2}$. Under our assumptions, the NED constants $d_{t}$ can be set equal to 1 , which implies that the mixingale constants $c_{t} \leq \max \left(\left\|X_{t}\right\|_{p+\delta}, 1\right)<\Delta<\infty$ for all $t$. Thus, $\left\|\max _{1 \leq i \leq \ell}\left|\sum_{s=j+i}^{j+\ell} X_{s}\right|\right\|_{2} \leq K \ell^{1 / 2}$, and from (18) we have that $k_{0}^{1 / 2} E\left(\sup _{r \in[0,1]}\left|T^{-1 / 2} \sum_{s=B+1}^{\ell} X_{j+s}\right|\right) \leq K$ uniformly in $j$, given that $k_{0}=T / \ell$. This completes the proof of (1). 
Next we show step (2). As in PP (2003), we consider the asymptotically equivalent statistic

$$
k_{0}^{-1 / 2} \sum_{m=1}^{\left[r k_{0}\right]+1} \lambda^{\prime} \Omega_{T}^{*-1 / 2}\left(\ell^{-1 / 2} \sum_{s=1}^{\ell}\left(X_{I_{m+s}}-E^{*} X_{I_{m+s}}\right)\right) \equiv k_{0}^{-1 / 2} \sum_{m=1}^{\left[r k_{0}\right]+1} V_{m}^{*},
$$

where $V_{m}^{*}=\lambda^{\prime} \Omega_{T}^{*-1 / 2} U_{m}^{*}$, with $U_{m}^{*}=\ell^{-1 / 2} \sum_{s=1}^{\ell}\left(X_{I_{m+s}}-E^{*} X_{I_{m+s}}\right)$. Note that $\left\{V_{m}^{*}: m=1, \ldots,\left[r k_{0}\right]+1\right\}$ is an array of independent variables with $E^{*}\left(V_{m}^{*}\right)=0$ and $\operatorname{Var}^{*}\left(V_{m}^{*}\right)=\lambda^{\prime} \Omega_{T}^{*-1 / 2} \operatorname{Var}^{*}\left(U_{m}^{*}\right) \Omega_{T}^{* 1 / 2} \lambda=$ 1 , where the last equality holds because we can show that $\operatorname{Var}^{*}\left(U_{m}^{*}\right)=\Omega_{T}^{*}$. We now apply a FCLT for martingale difference arrays (cf. Billingsley, 1968, p. 194). In particular, let $\xi_{T m}=\frac{1}{\sqrt{k_{0}}} V_{m}^{*}$ and note that $\xi_{T m}$ is a martingale array with respect to the $\sigma$-field $\mathcal{F}_{T, m-1}^{*}=\sigma\left(I_{1}, \ldots, I_{m-1}\right)$ given the independence of $V_{m}^{*}$. For each $r \in[0,1], \sum_{m=1}^{\left[r k_{0}\right]+1} \operatorname{Var}^{*}\left(\xi_{T m}\right)=\sum_{m=1}^{\left[r k_{0}\right]+1} \frac{1}{k_{0}}=\frac{\left[r k_{0}\right]+1}{k_{0}} \rightarrow r$ as $k_{0} \rightarrow \infty$, which verifies Billingsley's (1968, p. 194) condition (18.3). Next we verify that the Lindeberg condition (cf. Billingsley, 1968, eq. (18.4)) holds in probability. For this, it suffices that $\sum_{m=1}^{\left[k_{0} r\right]+1} E^{*}\left|\xi_{T m}\right|^{2+\delta} \rightarrow 0$ in probability. Since $\Omega_{T}^{*-1 / 2}=O_{P}(1)$, we need to show that $k_{0}^{-\frac{2+\delta}{2}} \sum_{m=1}^{\left[k_{0} r\right]+1} E^{*}\left|U_{m}^{*}\right|^{2+\delta} \rightarrow 0$ in probability. By definition of $U_{m}^{*}$, we have that

$$
E\left(E^{*}\left|U_{m}^{*}\right|^{2+\delta}\right) \leq K \ell^{-\frac{2+\delta}{2}} \frac{1}{T-\ell+1} \sum_{j=0}^{T-\ell} E\left(\left|\sum_{s=1}^{\ell} X_{j+s}\right|^{2+\delta}\right)
$$

An application of Lemma A.1 yields $E\left(\left|\sum_{s=1}^{\ell} X_{j+s}\right|^{2+\delta}\right) \leq K \ell^{\frac{2+\delta}{2}}$ uniformly in $j$, which implies that $E\left(E^{*}\left|U_{m}^{*}\right|^{2+\delta}\right)=O(1)$, showing that $k_{0}^{-\frac{2+\delta}{2}} \sum_{m=1}^{\left[k_{0} r\right]+1} E^{*}\left|U_{m}^{*}\right|^{2+\delta}=O_{P}\left(k_{0}^{-\delta / 2}\right)=o_{P}(1)$ since $k_{0}^{-\frac{\delta}{2}}=(\ell / T)^{\frac{\delta}{2}} \rightarrow 0$ under both sets of conditions on $\ell$.

Proof of Lemma A.4. As in the proof of Lemma A.3, we can write

$$
\begin{aligned}
T^{-1} \sum_{t=1}^{[r T]}\left(X_{t}^{*}-E^{*}\left(X_{t}^{*}\right)\right) & =T^{-1} \sum_{m=1}^{M_{r}} \sum_{s=1}^{\ell}\left(X_{I_{m}+s}-E^{*}\left(X_{I_{m}+s}\right)\right)+T^{-1} \sum_{s=B+1}^{\ell}\left(X_{I_{M_{r}}+s}-E^{*}\left(X_{I_{M_{r}}+s}\right)\right) \\
& \equiv A_{1 T}^{*}(r)+A_{2 T}^{*}(r) .
\end{aligned}
$$

Let $U_{m}^{*}=\sum_{s=1}^{\ell}\left(X_{I_{m}+s}-E^{*} X_{I_{m}+s}\right)$ and note that $S_{j}^{*}=\sum_{m=1}^{j} U_{m}^{*}$ is a martingale array with respect to $\mathcal{F}_{T, j}^{*}=\sigma\left(I_{1}, \ldots, I_{j}\right)$. Thus, by an application of Markov's inequality first, and of Doob's inequality second, we have that

$$
P^{*}\left(\sup _{r \in[0,1]}\left|A_{1 T}^{*}(r)\right|>\eta\right) \leq \frac{1}{\eta^{2} T^{2}} E^{*}\left(\sup _{r \in[0,1]}\left|\sum_{m=1}^{M_{r}} U_{m}^{*}\right|^{2}\right)=\frac{1}{\eta^{2} T^{2}} E^{*}\left(\max _{1 \leq j \leq k_{0}}\left|S_{j}^{*}\right|^{2}\right) \leq K T^{-2} E^{*}\left(\left|S_{k_{0}}^{*}\right|^{2}\right) .
$$

Adding and subtracting $\mu=E\left(X_{t}\right)$,

$$
E^{*}\left(\left|S_{k_{0}}^{*}\right|^{2}\right)=k_{0} E^{*}\left(U_{1}^{* 2}\right)=k_{0} E^{*}\left[\left(\sum_{s=1}^{\ell}\left(X_{I_{1}+s}-\mu-E^{*}\left(X_{I_{1}}-\mu\right)\right)\right)^{2}\right] \leq K k_{0} E^{*}\left(\left|\sum_{s=1}^{\ell}\left(X_{I_{1}+s}-\mu\right)\right|^{2}\right),
$$


for some constant $K$. Using the properties of the MBB and Lemma A.1, we can show that $E\left(E^{*}\left(\left|\sum_{s=1}^{\ell}\left(X_{I_{1}+s}-\mu\right)\right|^{2}\right)\right)=O(\ell)$, which implies $E^{*}\left(\left|S_{k_{0}}^{*}\right|^{2}\right)=O(T)$, and thus $P^{*}\left(\sup _{r \in[0,1]}\left|A_{1 T}^{*}(r)\right|>\eta\right)=O_{P}\left(T^{-1}\right)=o_{P}(1)$. Similarly, we can show that $E^{*}\left(\sup _{0 \leq r \leq 1}\left|A_{2 T}^{*}(r)\right|\right)=$ $O_{P}\left(\frac{\ell^{1 / 2}}{T}\right)=o_{P}(1)$ under both a) and b). 


\section{Appendix B}

This Appendix contains the proofs of the results in Section 5. We first present two useful lemmas.

We then present the proofs of the main results, followed by the proofs of the auxiliary lemmas. Throughout this Appendix, we let $\Omega=\pi^{2}(1) \sigma^{2}, \Lambda=\pi(1) \sigma$, and $\tilde{W}(r) \equiv \Lambda W_{1}(r)$.

Lemma A.5 Under Assumption 2, and for any fixed $b \in(0,1]$, we have

a) $T^{-1 / 2} \sum_{t=1}^{T} u_{t}=\Lambda W_{1}(1)+O_{P}\left(T^{-1 / 2+1 / p}\right)$.

b) $T^{-2} \sum_{t=1}^{T-1} S_{t}^{2}=\Omega \int_{0}^{1} W_{1}^{2}(r) d r+O_{P}\left(T^{-1 / 2+1 / p}\right)$.

c) $T^{-3 / 2} \sum_{t=1}^{T-1}\left(\frac{t}{T}\right) S_{t}=\Lambda \int_{0}^{1} r W_{1}(r) d r+O_{P}\left(T^{-1 / 2+1 / p}\right)$.

d) $T^{-3 / 2} \sum_{t=1}^{T-[b T]-1}\left(\frac{t}{T}\right) S_{t}=\Lambda \int_{0}^{1-b} r W_{1}(r) d r+O_{P}\left(T^{-1 / 2+1 / p}\right)$.

e) $T^{-3 / 2} \sum_{t=1}^{T-[b T]-1}\left(\frac{t}{T}\right) S_{t+[b T]}=\Lambda \int_{0}^{1-b} r W_{1}(r+b) d r+O_{P}\left(T^{-1 / 2+1 / p}\right)$.

f) $T^{-3 / 2} \sum_{t=1}^{T-[b T]-1} \frac{[b T]}{T} S_{t}=b \Lambda \int_{0}^{1-b} W_{1}(r) d r+O_{P}\left(T^{-1 / 2+1 / p}\right)$.

g) $T^{-2} \sum_{t=1}^{T-[b T]-1} S_{t} S_{t+[b T]}=\Omega \int_{0}^{1-b} W_{1}(r) W_{1}(r+b) d r+O_{P}\left(T^{-1 / 2+1 / p}\right)$.

Lemma A.6 Under Assumption 2, with probability approaching one, we have that

a) $T^{-1 / 2} \sum_{t=1}^{T} u_{t}^{*}=\Lambda^{*} W_{1}(1)+O_{P^{*}}\left(T^{-1 / 2+1 / p}\right)$.

b) $T^{-2} \sum_{t=1}^{T-1} S_{t}^{* 2}=\Omega^{*} \int_{0}^{1} W_{1}^{2}(r) d r+O_{P^{*}}\left(T^{-1 / 2+1 / p}\right)$.

c) $T^{-3 / 2} \sum_{t=1}^{T-1}\left(\frac{t}{T}\right) S_{t}^{*}=\Lambda^{*} \int_{0}^{1} r W_{1}(r) d r+O_{P^{*}}\left(T^{-1 / 2+1 / p}\right)$.

d) $T^{-3 / 2} \sum_{t=1}^{T-[b T]-1}\left(\frac{t}{T}\right) S_{t}^{*}=\Lambda^{*} \int_{0}^{1-b} r W_{1}(r) d r+O_{P^{*}}\left(T^{-1 / 2+1 / p}\right)$. 
e) $T^{-3 / 2} \sum_{t=1}^{T-[b T]-1}\left(\frac{t}{T}\right) S_{t+[b T]}^{*}=\Lambda^{*} \int_{0}^{1-b} r W_{1}(r+b) d r+O_{P^{*}}\left(T^{-1 / 2+1 / p}\right)$.

f) $T^{-3 / 2} \sum_{t=1}^{T-[b T]-1} \frac{[b T]}{T} S_{t}^{*}=b \Lambda^{*} \int_{0}^{1-b} W_{1}(r) d r+O_{P^{*}}\left(T^{-1 / 2+1 / p}\right)$.

g) $T^{-2} \sum_{t=1}^{T-[b T]-1} S_{t}^{*} S_{t+[b T]}^{*}=\Omega^{*} \int_{0}^{1-b} W_{1}(r) W_{1}(r+b) d r+O_{P^{*}}\left(T^{-1 / 2+1 / p}\right)$.

where $\Omega^{*}=\sigma^{2} \sum_{i=1}^{\infty} \pi_{i}^{2}$ and $\Lambda^{*}=\Omega^{* 1 / 2}$.

Proof of Lemma 5.1. Theorem 3 of Akonom (1993) implies that under our assumptions

$$
P\left(\sup _{r \in[0,1]}\left|W_{T}(r)-\tilde{W}(r)\right|>c_{T}\right) \leq C_{2} T^{1-p / 2} c_{T}^{-p} E\left|\varepsilon_{t}\right|^{p},
$$

for any sequence $c_{T}$ such that $T^{-1 / 2+1 / p} \leq c_{T} \leq C_{1}(\log T)^{1 / 2}$, where $C_{1}$ and $C_{2}$ are constants independent of $T$. Part a) follows by letting $c_{T}=c T^{-1 / 2+1 / p}$, for some constant $c$, whereas part b) follows by setting $c_{T}=c T^{-1 / 2+3 / 2 p}$.

Proof of Lemma 5.2, Write $\hat{\Omega}=J_{1}-J_{2}$, with $J_{1}=2 b^{-1} T^{-2} \sum_{t=1}^{T-1} \hat{S}_{t}^{2}$ and $J_{2}=2 b^{-1} T^{-2} \sum_{t=1}^{T-[b T]-1} \hat{S}_{t} \hat{S}_{t+[b T]}$. We can write $J_{1}=2 I_{1}-4 I_{2}+2 I_{3}$, where by Lemma A.5, $I_{1}=T^{-2} \sum_{t=1}^{T-1} S_{t}^{2}=\Omega \int_{0}^{1} W_{1}^{2}(r) d r+$ $O_{P}\left(T^{-1 / 2+1 / p}\right)$;

$$
\begin{aligned}
I_{2} & =T^{-2} \sum_{t=1}^{T-1}\left(\frac{t}{T}\right) S_{t} S_{T}=\left(T^{-3 / 2} \sum_{t=1}^{T-1}\left(\frac{t}{T}\right) S_{t}\right)\left(T^{-1 / 2} S_{T}\right) \\
& =\left(\Lambda \int_{0}^{1} r W_{1}(r) d r+O_{P}\left(T^{-1 / 2+1 / p}\right)\right)\left(\Lambda W_{1}(1)+O_{P}\left(T^{-1 / 2+1 / p}\right)\right) \\
& =\Omega \int_{0}^{1} r W_{1}(r) W_{1}(1) d r+O_{P}\left(T^{-1 / 2+1 / p}\right)
\end{aligned}
$$

and

$$
\begin{aligned}
I_{3} & =T^{-2} \sum_{t=1}^{T-1}\left(\frac{t}{T}\right)^{2} S_{T}^{2}=T^{-1} \sum_{t=1}^{T-1}\left(\frac{t}{T}\right)^{2}\left(T^{-1 / 2} S_{T}\right)^{2} \\
& =\left(\frac{1}{3}+O\left(T^{-1}\right)\right)\left(\Lambda W_{1}(1)+O_{P}\left(T^{-1 / 2+1 / p}\right)\right)^{2}=\frac{1}{3} \Omega W_{1}^{2}(1)+O_{P}\left(T^{-1 / 2+1 / p}\right)
\end{aligned}
$$

since $T^{-1} \sum_{t=1}^{T-1}\left(\frac{t}{T}\right)^{2}=\frac{1}{6} \frac{2 T^{2}-3 T+1}{T^{2}}=\frac{1}{3}+O\left(T^{-1}\right)$. Thus,

$$
J_{1}=\Omega\left[\frac{2}{b} \int_{0}^{1}\left(W_{1}(r)-r W_{1}(1)\right)^{2} d r\right]+O_{P}\left(T^{-1 / 2+1 / p}\right) .
$$


Next we analyze $J_{2}$. Notice that we can write

$$
\begin{aligned}
\hat{S}_{t} \hat{S}_{t+[b T]} & =\left(S_{t}-\frac{t}{T} S_{T}\right)\left(S_{t+[b T]}-\frac{t+[b T]}{T} S_{T}\right) \\
& =S_{t} S_{t+[b T]}-\frac{t+[b T]}{T} S_{t} S_{T}-\frac{t}{T} S_{t+[b T]} S_{T}+\frac{t}{T} \frac{t+[b T]}{T} S_{T}^{2},
\end{aligned}
$$

implying that

$$
\begin{aligned}
J_{2}= & \frac{2}{b} T^{-2} \sum_{t=1}^{T-[b T]-1} S_{t} S_{t+[b T]}-\frac{2}{b} T^{-2} \sum_{t=1}^{T-[b T]-1} \frac{t+[b T]}{T} S_{t} S_{T} \\
& -\frac{2}{b} T^{-2} \sum_{t=1}^{T-[b T]-1} \frac{t}{T} S_{t+[b T]} S_{T}+\frac{2}{b} T^{-2} \sum_{t=1}^{T-[b T]-1} \frac{t}{T} \frac{t+[b T]}{T} S_{T}^{2} \\
\equiv & A_{1}-A_{2}-A_{3}+A_{4} .
\end{aligned}
$$

By Lemma A.5, we have that

$$
\begin{aligned}
& A_{1}=\frac{2}{b} \Omega \int_{0}^{1-b} W_{1}(r) W_{1}(r+b) d r+O_{P}\left(T^{-1 / 2+1 / p}\right) \\
& A_{2}= \frac{2}{b} T^{-2} \sum_{t=1}^{T-[b T]-1} \frac{t}{T} S_{t} S_{T}+\frac{2}{b} T^{-2} \sum_{t=1}^{T-[b T]-1} \frac{[b T]}{T} S_{t} S_{T} \\
&= \frac{2}{b}\left(T^{-1 / 2} S_{T}\right)\left(T^{-3 / 2} \sum_{t=1}^{T-[b T]-1}\left(\frac{t}{T}\right) S_{t}\right)+\frac{2}{b}\left(T^{-1 / 2} S_{T}\right)\left(T^{-3 / 2} \sum_{t=1}^{T-[b T]-1} \frac{[b T]}{T} S_{t}\right) \\
&= \frac{2}{b} \Omega \int_{0}^{1-b} r W_{1}(r) W_{1}(1) d r+\frac{2}{b} b \Omega \int_{0}^{1-b} W_{1}(r) W_{1}(1) d r+O_{P}\left(T^{-1 / 2+1 / p}\right) \\
&= \frac{2}{b} \Omega \int_{0}^{1-b}(r+b) W_{1}(r) W_{1}(1) d r+O_{P}\left(T^{-1 / 2+1 / p}\right) ; \\
& A_{3}=\frac{2}{b}\left(T^{-1 / 2} S_{T}\right)\left(T^{-3 / 2} \sum_{t=1}^{T-[b T]-1} \frac{t}{T} S_{t+[b T]}\right)=\frac{2}{b} \Omega \int_{0}^{1-b} r W_{1}(r+b) W_{1}(1) d r+O_{P}\left(T^{-1 / 2+1 / p}\right),
\end{aligned}
$$

and

$$
\begin{aligned}
A_{4} & =\frac{2}{b}\left(T^{-1 / 2} S_{T}\right)^{2}\left(T^{-1} \sum_{t=1}^{T-[b T]} \frac{-1}{T} \frac{t+[b T]}{T}\right) \\
& =\frac{2}{b} \Omega W_{1}^{2}(1)\left[\frac{1}{3}(1-b)^{3}+\frac{1}{2} b(1-b)^{2}\right]+O_{P}\left(T^{-1 / 2+1 / p}\right) .
\end{aligned}
$$


The last result uses the fact that

$$
\begin{aligned}
T^{-3} \sum_{t=1}^{T-[b T]-1} t(t+[b T]) & =T^{-3} \sum_{t=1}^{T-[b T]-1} t^{2}+T^{-3} \sum_{t=1}^{T-[b T]-1} t[b T] \\
& =\frac{1}{3}(1-b)^{3}+\frac{1}{2} b(1-b)^{2}+O\left(T^{-1}\right) .
\end{aligned}
$$

The desired result follows from combining all the previous expansions.

Proof of Theorem [5.1. Write $t_{\hat{\beta}_{1}}=P_{T} \Omega_{1}^{-1 / 2}\left(\frac{\hat{\Omega}}{\Omega_{1}}\right)^{-1 / 2}$, where $P_{T}=T^{-1 / 2} \sum_{t=1}^{T} u_{t}$ and $\Omega_{1}=$ $\Omega Q_{1}(b)$ is the leading term of the expansion of $\hat{\Omega}$. Note that by a Taylor expansion of $f(x)=$ $(1+x)^{-1 / 2}$ around 0 we can write

$$
\left(\frac{\hat{\Omega}}{\Omega_{1}}\right)^{-1 / 2}=\left(1+\frac{\hat{\Omega}-\Omega_{1}}{\Omega_{1}}\right)^{-1 / 2}=1-\frac{1}{2} \frac{\hat{\Omega}-\Omega_{1}}{\Omega_{1}}+O_{P}\left(\left(\hat{\Omega}-\Omega_{1}\right)^{2}\right) .
$$

Lemma 5.2 implies that $\hat{\Omega}-\Omega_{1}=O_{P}\left(T^{-1 / 2+1 / p}\right)$, and since $\Omega_{1}=O_{P}(1)$, we get that

$$
t_{\hat{\beta}_{1}}=P_{T} \Omega_{1}^{-1 / 2}\left(1+O_{P}\left(T^{-1 / 2+1 / p}\right)\right)=P_{T} \Omega_{1}^{-1 / 2}+O_{P}\left(T^{-1 / 2+1 / p}\right) .
$$

Lemma A.5. a) now implies the result.

Proof of Corollary [5.1. We follow the proof of Corollary 3.8 of Park (2003). In particular, the result follows from Lemma A4 of Park (2003) given that the error terms of the asymptotic expansions in Lemma A.5 are distributionally of order $O\left(T^{-a}\right)$, with $a=1 / 2-3 /(2 p)$, and that the density of $\Omega_{1}^{-1}$ is bounded and all its moments are finite (which follows because $\Omega_{1}$ is a quadratic form of a Brownian motion with a truncated positive definite kernel). The remainder terms for each statistic are defined in the proof of Lemma A.5. Thus, the remainder term of part a) of Lemma A.5 is equal to $R_{1 T}=W_{T}(1)-\tilde{W}(1)$, which is distributionally of order $O\left(T^{-a}\right)$ given part b) of Lemma 5.1. For part b), the remainder is $R_{2 T}=R_{2 T}^{(1)}+R_{2 T}^{(2)}$, where $\left|R_{2 T}^{(1)}\right| \leq \sup \left|W_{T}(r)-\tilde{W}(r)\right|^{2}$ and $\left|R_{2 T}^{(2)}\right| \leq 2\left(\sup \left|W_{T}(r)-\tilde{W}(r)\right|^{2}\right)^{1 / 2}\left(\int_{0}^{1} \tilde{W}^{2}(r) d r\right)^{1 / 2}$. We have that

$$
\begin{aligned}
P\left(\sup \left|W_{T}(r)-\tilde{W}(r)\right|^{2} \geq T^{-a}\right) & \leq P\left(\sup \left|W_{T}(r)-\tilde{W}(r)\right|^{2} \geq T^{-2 a}\right) \\
& \leq P\left(\sup \left|W_{T}(r)-\tilde{W}(r)\right| \geq T^{-a}\right)=O\left(T^{-a}\right),
\end{aligned}
$$

showing that $R_{2 T}^{(1)}$ is distributionally of order $O\left(T^{-a}\right)$. Since $\int_{0}^{1} \tilde{W}^{2}(r) d r$ has moments finite up to any order, Lemma A4. b) of Park (2003) implies that $R_{2 T}^{(2)}$ is also distributionally of order $O\left(T^{-a}\right)$. For part c), the remainder is $R_{3 T}=R_{3 T}^{(1)}+R_{3 T}^{(2)}$, where $\left|R_{3 T}^{(1)}\right| \leq \sup \left|W_{T}(r)-\tilde{W}(r)\right|$ and $\left|R_{3 T}^{(2)}\right| \leq \sup \left|W_{T}(r)-\tilde{W}(r)\right|+\frac{1}{T} \int_{0}^{1}|\tilde{W}(r)| d r$. Since $\sup \left|W_{T}(r)-\tilde{W}(r)\right|$ is distributionally of order $O\left(T^{-a}\right)$ by Lemma[5.1 b), we only need to show that the same is true for $\frac{1}{T} \int_{0}^{1}|\tilde{W}(r)| d r$. This follows by an application of Markov's inequality, given that $E\left|\int_{0}^{1}\right| \tilde{W}(r)|d r|<\infty$. For part d), note 
that the remainder is $R_{4 T}=R_{4 T}^{(1)}+R_{4 T}^{(2)}$, where $R_{4 T}^{(1)}$ is majorized by the same term as $R_{3 T}$, whereas $\left|R_{4 T}^{(2)}\right| \leq \sup \left|W_{T}(r)-\tilde{W}(r)\right|+\frac{1}{T} \sup |\tilde{W}(r)|$, which can be handled as $R_{3 T}^{(2)}$. The remainder in part e) can be decomposed as $R_{5 T}^{(1)}+R_{5 T}^{(2)}+R_{5 T}^{(3)}+R_{5 T}^{(4)}+R_{5 T}^{(5)}+R_{5 T}^{(6)}$, where $R_{5 T}^{(1)}$ and $R_{5 T}^{(5)}$ are majorized by $\sup \left|W_{T}(r)-\tilde{W}(r)\right| ; R_{5 T}^{(2)}$ and $R_{5 T}^{(6)}$ are majorized by $\sup \left|W_{T}(r)-\tilde{W}(r)\right|+\frac{1}{T} \int_{0}^{1}|\tilde{W}(r)| d r$. For $R_{5 T}^{(3)}$ and $R_{5 T}^{(4)}$ we have that for $i=3,4$,

$T^{a} P\left(\left|R_{5 T}^{(i)}\right| \geq T^{-a}\right) \leq T^{a} P\left(\left|T^{-3 / 2} S_{[b T]}\right| \geq T^{-a}\right) \leq T^{a} \frac{E\left|S_{[b T]}\right|^{p}}{T^{(3 / 2-a) p}} \leq K T^{a+p / 2-(3 / 2-a) p}=K T^{-1-\frac{3}{2 p}-\frac{p}{2}}$,

which is $o(1)$ for any $p>0$ and where we have used the fact that $E\left|S_{[b T]}\right|^{p} \leq T^{p / 2}$ by Lemma A.1, part b). The remainders in parts f) and e) can be analyzed using similar arguments and therefore we omit their proofs.

Proof of Lemma 5.3. Since $u_{t}^{*}$ are i.i.d. we can apply the strong approximation result of Sakhanenko (1980) to $W_{T}^{*}$, as in the proof of Lemma 2.4 of Park (2006). That is, we may choose $W_{T}^{*}$ in the same probability space as the Brownian motion $\tilde{W}^{*}(r)=\Omega^{* 1 / 2} W_{1}(r)$ such that $W_{T}^{*}$ has the same conditional distribution as $W_{T}^{0 *}$ and verifies the following condition:

$$
P^{*}\left(\sup _{0 \leq r \leq 1}\left|W_{T}^{*}(r)-\tilde{W}^{*}(r)\right|>c_{T}\right) \leq K c_{T}^{-p} T^{1-p / 2} E^{*}\left|u_{t}^{*}\right|^{p},
$$

where $\tilde{W}^{*}(r)=\Omega^{* 1 / 2} W(r)$. If we show that $E^{*}\left|u_{t}^{*}\right|^{p}=O_{P}(1)$, the first result follows by letting $c_{T}=c T^{-1 / 2+1 / p}$ for some large $c>0$ whereas the second result follows by letting $c_{T}=$ $c T^{-1 / 2+3 /(2 p)}$. Note that

$$
E^{*}\left|u_{t}^{*}\right|^{p}=T^{-1} \sum_{t=1}^{T}\left|\hat{u}_{t}\right|^{p}=T^{-1} \sum_{t=1}^{T}\left|u_{t}-\bar{u}\right|^{p} \leq K T^{-1} \sum_{t=1}^{T}\left|u_{t}\right|^{p} \rightarrow K E\left|u_{t}\right|^{p}<\infty,
$$

for some constant $K$, in probability. This proves that $E^{*}\left|u_{t}^{*}\right|^{p}=O_{P}(1)$.

Proof of Lemma 5.4. Given Lemma 5.3, the proof follows the same reasoning as that of Lemma 5.2 ,

Proof of Theorem 5.2. Given Lemma 5.3, the proof follows the same reasoning as that of Theorem 5.1 .

Proof of Corollary 5.2. Given part b) of Lemma 5.3 and Theorem 5.2, the proof is analogous to that of Corollary 5.2 ,

Proof of Lemma A.5. We follow closely the proof of Lemma 3.1 of Park (2006). For a), note that $T^{-1 / 2} \sum_{t=1}^{T} u_{t}=W_{T}(1)=\tilde{W}(1)+R_{1 T}$, where $R_{1 T}=W_{T}(1)-\tilde{W}(1)$. By Lemma 5.1. a), $R_{1 T}=O_{P}\left(T^{-1 / 2+1 / p}\right)$, proving the result. For b), let $S_{0} \equiv 0$ and write $T^{-2} \sum_{t=1}^{T-1} S_{t}^{2}=$ $T^{-2} \sum_{t=1}^{T} S_{t-1}^{2}$. Note that $T^{-1 / 2} S_{t-1}=T^{-1 / 2} \sum_{i=1}^{t-1} u_{i}=W_{T}\left(\frac{t-1}{T}\right)$. Thus,

$$
T^{-2} \sum_{t=1}^{T} S_{t-1}^{2}=T^{-1} \sum_{t=1}^{T} W_{T}^{2}\left(\frac{t-1}{T}\right)=\int_{0}^{1} W_{T}^{2}(r) d r=\int_{0}^{1} \tilde{W}^{2}(r) d r+R_{2 T},
$$


where $R_{2 T}=\int_{0}^{1}\left(W_{T}^{2}(r)-\tilde{W}^{2}(r)\right) d r$. We can write

$$
R_{2 T}=\int_{0}^{1}\left(W_{T}(r)-\tilde{W}(r)\right)^{2} d r+2 \int_{0}^{1}\left(W_{T}(r)-\tilde{W}(r)\right) \tilde{W}(r) d r \equiv R_{2 T}^{(1)}+R_{2 T}^{(2)} .
$$

Then, by Lemma 5.1.a),

$$
R_{2 T}^{(1)} \leq \int_{0}^{1} \sup _{r \in[0,1]}\left|W_{T}(r)-\tilde{W}(r)\right|^{2} d r \leq\left(\sup _{r \in[0,1]}\left|W_{T}(r)-\tilde{W}(r)\right|\right)^{2}=O_{P}\left(\left(T^{-1 / 2+1 / p}\right)^{2}\right) .
$$

For $R_{2 T}^{(2)}$, by the Cauchy-Schwartz inequality,

$$
\left|R_{2 T}^{(2)}\right| \leq 2\left(\int_{0}^{1}\left(W_{T}(r)-\tilde{W}(r)\right)^{2} d r\right)^{1 / 2}\left(\int_{0}^{1} \tilde{W}^{2}(r) d r\right)^{1 / 2}=2 A_{1 T} \cdot A_{2 T} .
$$

By Lemma 5.1.a), $A_{1 T}=O_{P}\left(T^{-1 / 2+1 / p}\right)$ and $A_{2 T}=\int_{0}^{1} \tilde{W}^{2}(r) d r=O_{P}(1)$. For c), we can write

$$
\begin{aligned}
T^{-3 / 2} \sum_{t=1}^{T-1}\left(\frac{t}{T}\right) S_{t} & =T^{-1} \sum_{t=1}^{T} \frac{t-1}{T}\left(T^{-1 / 2} S_{t-1}\right)=T^{-1} \sum_{t=1}^{T} \frac{t-1}{T} W_{T}\left(\frac{t-1}{T}\right) \\
& =\sum_{t=1}^{T} \int_{(t-1) / T}^{t / T} \frac{[T r]}{T} W_{T}(r) d r=\int_{0}^{1} \frac{[T r]}{T} W_{T}(r) d r=\int_{0}^{1} r \tilde{W}(r) d r+R_{3 T},
\end{aligned}
$$

where $R_{3 T}=R_{3 T}^{(1)}+R_{3 T}^{(2)}$, with

$$
R_{3 T}^{(1)}=\int_{0}^{1} r\left(W_{T}(r)-\tilde{W}(r)\right) d r \quad \text { and } \quad R_{3 T}^{(2)}=\int_{0}^{1}\left(\frac{[T r]}{T}-r\right) W_{T}(r) d r .
$$

By Lemma 5.1, a) we have that $R_{3 T}^{(1)}=O_{P}\left(T^{-1 / 2+1 / p}\right)$. For $R_{3 T}^{(2)}$, note that

$$
\begin{aligned}
\left|R_{3 T}^{(2)}\right| & \leq \int_{0}^{1}\left|\frac{[T r]}{T}-r\right|\left|W_{T}(r)\right| d r \leq \frac{1}{T} \int_{0}^{1}\left|W_{T}(r)\right| d r \\
& =\frac{1}{T} \int_{0}^{1}\left|W_{T}(r)-\tilde{W}(r)\right| d r+\frac{1}{T} \int_{0}^{1}|\tilde{W}(r)| d r \equiv A_{3 T}+A_{4 T},
\end{aligned}
$$

where $A_{3 T}=O_{P}\left(T^{-3 / 2+1 / p}\right)$ and $A_{4 T}=O_{P}\left(T^{-1}\right)=O_{P}\left(T^{-1 / 2+1 / p}\right)$. For d), we have

$$
T^{-3 / 2} \sum_{t=1}^{T-[b T]-1} \frac{t}{T} S_{t}=\int_{0}^{1-b} r \tilde{W}(r) d r+R_{4 T},
$$

where $R_{4 T} \equiv R_{4 T}^{(1)}+R_{4 T}^{(2)}$. $\quad R_{4 T}^{(1)}$ is of the same form as $R_{3 T}$ but with the $\int_{0}^{1}$ replaced by $\int_{0}^{1-b}$, and $R_{4 T}^{(2)}=\int_{1-b}^{1-b+b-[b T] / T} \frac{[T r]}{T} W_{T}(r) d r$. Following the proof for $R_{3 T}$, we can show that $R_{4 T}^{(1)}=$ 
$O_{P}\left(T^{-1 / 2+1 / p}\right) . R_{4 T}^{(2)}$ can be bounded by

$$
\begin{gathered}
\int_{1-b}^{1-b+b-[b T] / T}\left|\frac{[T r]}{T}\right|\left|W_{T}(r)\right| d r \leq \int_{1-b}^{1-[b T] / T}|r|\left|W_{T}(r)\right| d r \\
\leq \sup _{r \in[0,1]}\left|W_{T}(r)\right| \int_{1-b}^{1-[b T] / T} 1 d r \leq \sup _{r \in[0,1]}\left|W_{T}(r)\right|\left|b-\frac{[b T]}{T}\right|=O_{P}\left(T^{-1}\right),
\end{gathered}
$$

since $\sup _{r \in[0,1]}\left|W_{T}(r)\right|=O_{P}(1)$ and $\left|b-\frac{[b T]}{T}\right|=O\left(T^{-1}\right)$. For e), write

$$
\begin{aligned}
T^{-3 / 2} \sum_{t=1}^{T-[b T]-1} \frac{t}{T} S_{t+[b T]} & =T^{-1} \sum_{t=1}^{T-[b T]} \frac{t-1}{T}\left(T^{-1 / 2} S_{t+[b T]-1}\right)=T^{-1} \sum_{t=[b T]+1}^{T} \frac{t-1-[b T]}{T}\left(T^{-1 / 2} S_{t-1}\right) \\
& =T^{-1} \sum_{t=[b T]+1}^{T} \frac{t-1}{T}\left(T^{-1 / 2} S_{t-1}\right)-T^{-1} \sum_{t=[b T]+1}^{T} \frac{[b T]}{T}\left(T^{-1 / 2} S_{t-1}\right) \equiv M_{1 T}+M_{2 T} .
\end{aligned}
$$

We analyze $M_{1 T}$ and $M_{2 T}$ separately. For $M_{1 T}$, we can write

$$
\begin{aligned}
M_{1 T} & =\int_{[b T] / T}^{1} \frac{[T r]}{T} W_{T}(r) d r=\int_{b}^{1} \frac{[T r]}{T} W_{T}(r) d r+\int_{[b T] / T}^{b} \frac{[T r]}{T} W_{T}(r) d r \\
& =\int_{b}^{1} r \tilde{W}(r) d r+\int_{b}^{1} r\left(W_{T}(r)-\tilde{W}(r)\right) d r+\int_{b}^{1}\left(\frac{[r T]}{T}-r\right) W_{T}(r) d r+\int_{[b T] / T}^{b} \frac{[T r]}{T} W_{T}(r) d r \\
& \equiv \int_{b}^{1} r \tilde{W}(r) d r+R_{5 T}^{(1)}+R_{5 T}^{(2)}+R_{5 T}^{(3)} .
\end{aligned}
$$

We can majorize $R_{5 T}^{(1)}$ and $R_{5 T}^{(2)}$ by the same terms that majorize $R_{3 T}^{(1)}$ and $R_{3 T}^{(2)}$ respectively. For $R_{5 T}^{(3)}$ we have that

$$
\left|R_{5 T}^{(3)}\right| \leq\left|T^{-1 / 2} S_{[b T]}\right|\left(b-\frac{[b T]}{T}\right) \leq\left|T^{-3 / 2} S_{[b T]}\right|=O_{P}\left(T^{-1 / 2+1 / p}\right) .
$$

For $M_{2 T}$, we can write

$$
\begin{aligned}
M_{2 T} & =-b \int_{b}^{1} \tilde{W}(r) d r-\frac{[b T]}{T} \int_{[b T] / T}^{b} W_{T}(r) d r-b \int_{b}^{1}\left(W_{T}(r)-\tilde{W}(r)\right) d r-\left(\frac{[b T]}{T}-b\right) \int_{b}^{1} W_{T}(r) d r \\
& \equiv-b \int_{b}^{1} \tilde{W}(r) d r-R_{5 T}^{(4)}-R_{5 T}^{(5)}-R_{5 T}^{(6)} .
\end{aligned}
$$

We can show that $\left|R_{5 T}^{(4)}\right| \leq\left|T^{-3 / 2} S_{[b T]}\right|=O_{P}\left(T^{-1 / 2+1 / p}\right)$ and $R_{5 T}^{(5)}$ and $R_{5 T}^{(6)}$ can also be shown to be $O_{P}\left(T^{-1 / 2+1 / p}\right)$ by arguments similar to those used above. Thus

$$
M_{1 T}+M_{2 T}=\int_{b}^{1}(r-b) \tilde{W}(r) d r+O_{P}\left(T^{-1 / 2+1 / p}\right)=\int_{0}^{1-b} r \tilde{W}(r+b) d r+O_{P}\left(T^{-1 / 2+1 / p}\right) .
$$


For f), letting $S_{0} \equiv 0$, we can write

$$
\begin{aligned}
T^{-3 / 2} \sum_{t=1}^{T-[b T]-1} \frac{[b T]}{T} S_{t} & =T^{-1} \sum_{t=1}^{T-[b T]} \frac{[b T]}{T}\left(T^{-1 / 2} S_{t-1}\right)=\frac{[b T]}{T} \sum_{t=1}^{T-[b T]} T^{-1} W_{T}\left(\frac{t-1}{T}\right) \\
& =\frac{[b T]}{T} \int_{0}^{1-b} W_{T}(r) d r+\frac{[b T]}{T} \int_{1-b}^{(T-[b T]) / T} W_{T}(r) d r \\
& =\frac{[b T]}{T} \int_{0}^{1-b} \tilde{W}(r) d r+\frac{[b T]}{T} \int_{0}^{1-b}\left(W_{T}(r)-\tilde{W}(r)\right) d r+\frac{[b T]}{T} \int_{1-b}^{(T-[b T]) / T} W_{T}(r) d r \\
& \equiv b \int_{0}^{1-b} \tilde{W}(r) d r+R_{6 T},
\end{aligned}
$$

where $R_{6 T}=R_{6 T}^{(1)}+R_{6 T}^{(2)}+R_{6 T}^{(3)}$, with

$$
\begin{aligned}
R_{6 T}^{(1)} & =\left(\frac{[b T]}{T}-b\right) \int_{0}^{1-b} \tilde{W}(r) d r=O_{P}\left(T^{-1}\right) \\
R_{6 T}^{(2)} & =\frac{[b T]}{T} \int_{0}^{1-b}\left(W_{T}(r)-\tilde{W}(r)\right) d r=O_{P}\left(T^{-1 / 2+1 / p}\right) ; \\
R_{6 T}^{(3)} & =\frac{[b T]}{T} \int_{1-b}^{(T-[b T]) / T} W_{T}(r) d r \leq \frac{1}{T} \int_{0}^{1}\left|W_{T}(r)\right| d r=O_{P}\left(T^{-1}\right) .
\end{aligned}
$$

Finally, for g) write

$$
\begin{aligned}
T^{-2} \sum_{t=1}^{T-[b T]-1} S_{t} S_{t+[b T]} & =T^{-1} \sum_{t=1}^{T-[b T]}\left(T^{-1 / 2} S_{t-1}\right)\left(T^{-1 / 2} S_{t-1+[b T]}\right) \\
& =T^{-1} \sum_{t=1+[b T]}^{T}\left(T^{-1 / 2} S_{t-1-[b T]}\right)\left(T^{-1 / 2} S_{t-1}\right) \\
& =\int_{b}^{1} \tilde{W}(r-b) \tilde{W}(r) d r+R_{7 T}=\int_{0}^{1-b} \tilde{W}(r) \tilde{W}(r+b) d r+R_{7 T}
\end{aligned}
$$

where $R_{7 T}=R_{7 T}^{(1)}+R_{7 T}^{(2)}+R_{7 T}^{(3)}$, with

$$
\begin{aligned}
R_{7 T}^{(1)} & =\int_{b}^{1}\left(W_{T}(r-b)-\tilde{W}(r-b)\right) \tilde{W}(r) d r \\
R_{7 T}^{(2)} & =\int_{b}^{1}\left(W_{T}(r)-\tilde{W}(r)\right) W_{T}(r-b) d r \\
R_{7 T}^{(3)} & =\int_{[b T] / T}^{b} W_{T}(r-b) W_{T}(r) d r .
\end{aligned}
$$

Using arguments similar as above, we can show that each of these terms is $O_{P}\left(T^{-1 / 2+1 / p}\right)$, completing the proof.

Proof of Lemma A.6. The first result follows trivially from part a) of Lemma 5.3. The remaining result follow exactly as in the proof of Lemma A.5. given Lemma 5.3 . 

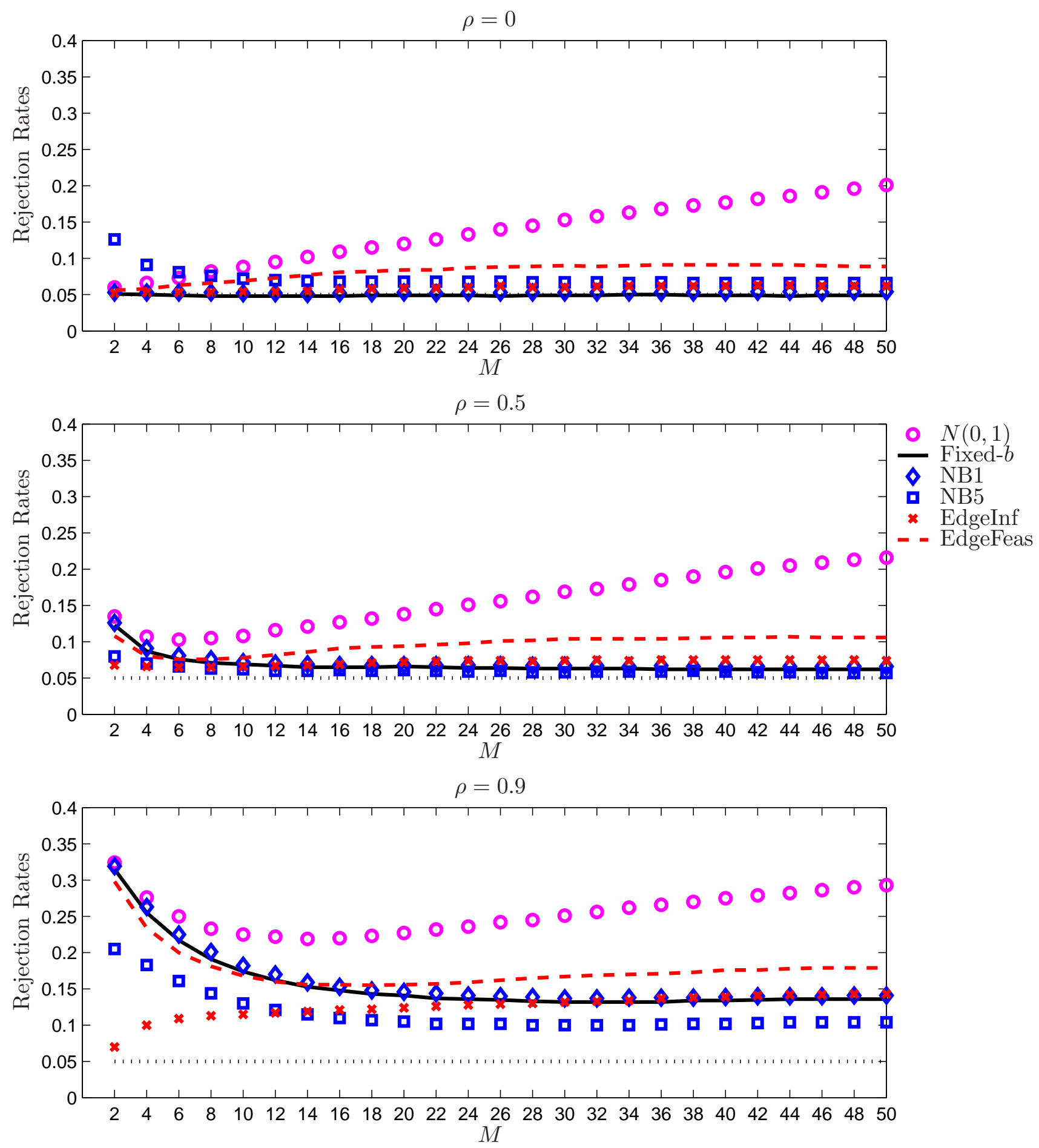

Figure 1: Empirical Null Rejection Probabilities, Simple Location Model, Bartlett Kernel, $T=50$ 

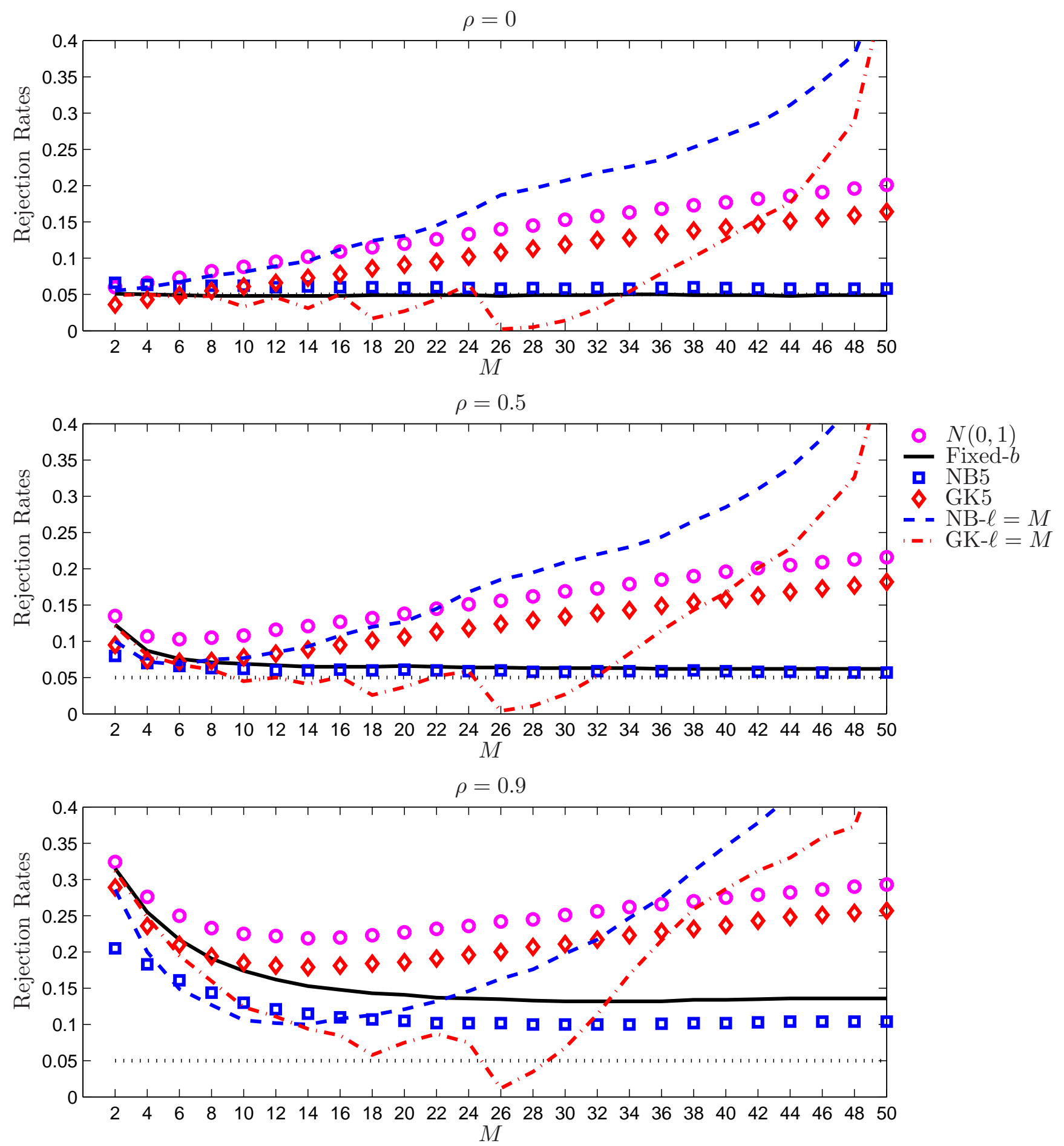

Figure 2: Empirical Null Rejection Probabilities, Simple Location Model, Bartlett Kernel, $T=50$ 

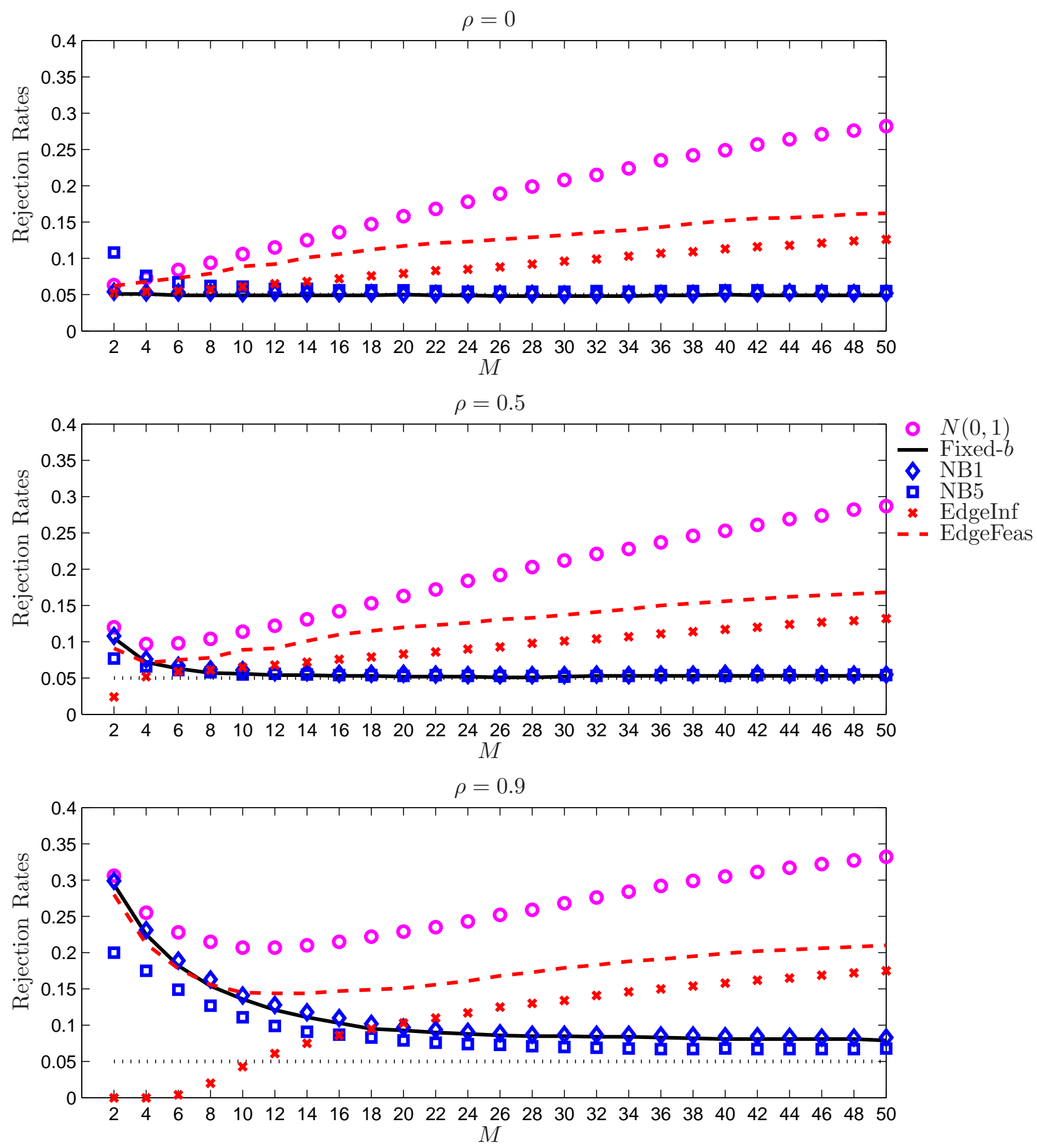

Figure 3: Empirical Null Rejection Probabilities, Simple Location Model, QS Kernel, $T=50$ 

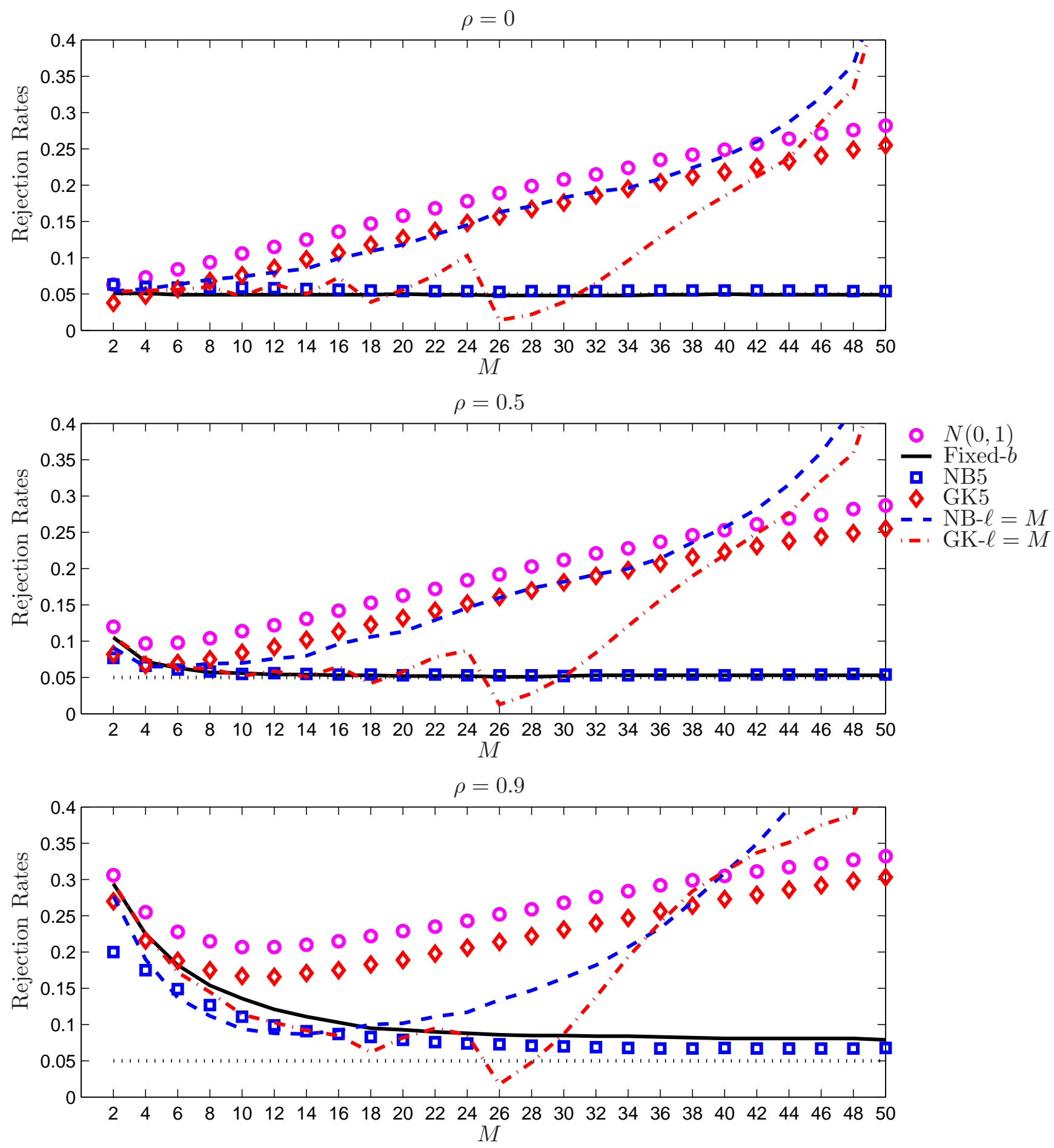

Figure 4: Empirical Null Rejection Probabilities, Simple Location Model, QS Kernel, $T=50$ 

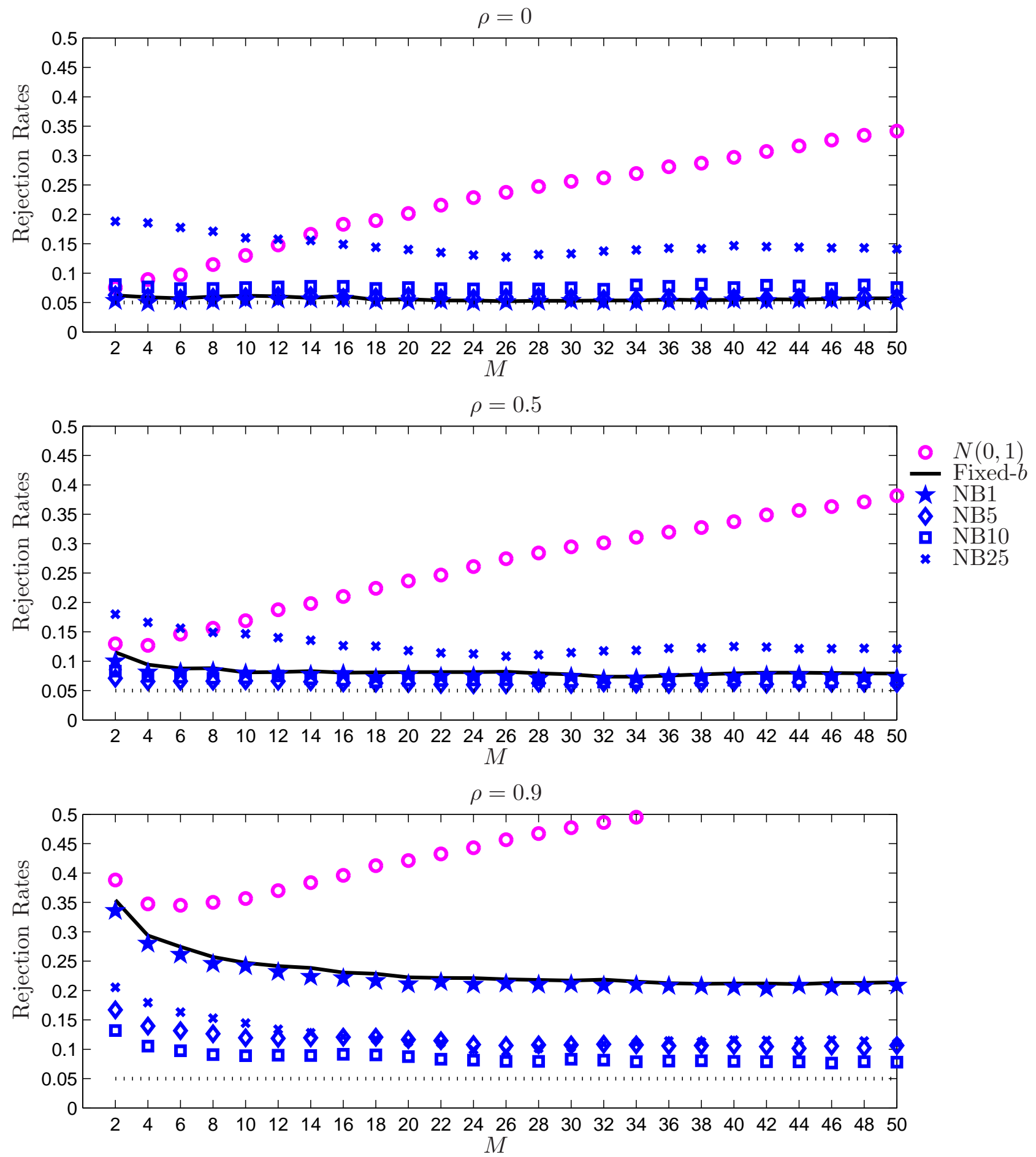

Figure 5: Empirical Null Rejection Probabilities, Linear Regression, Bartlett Kernel, $T=50$ 

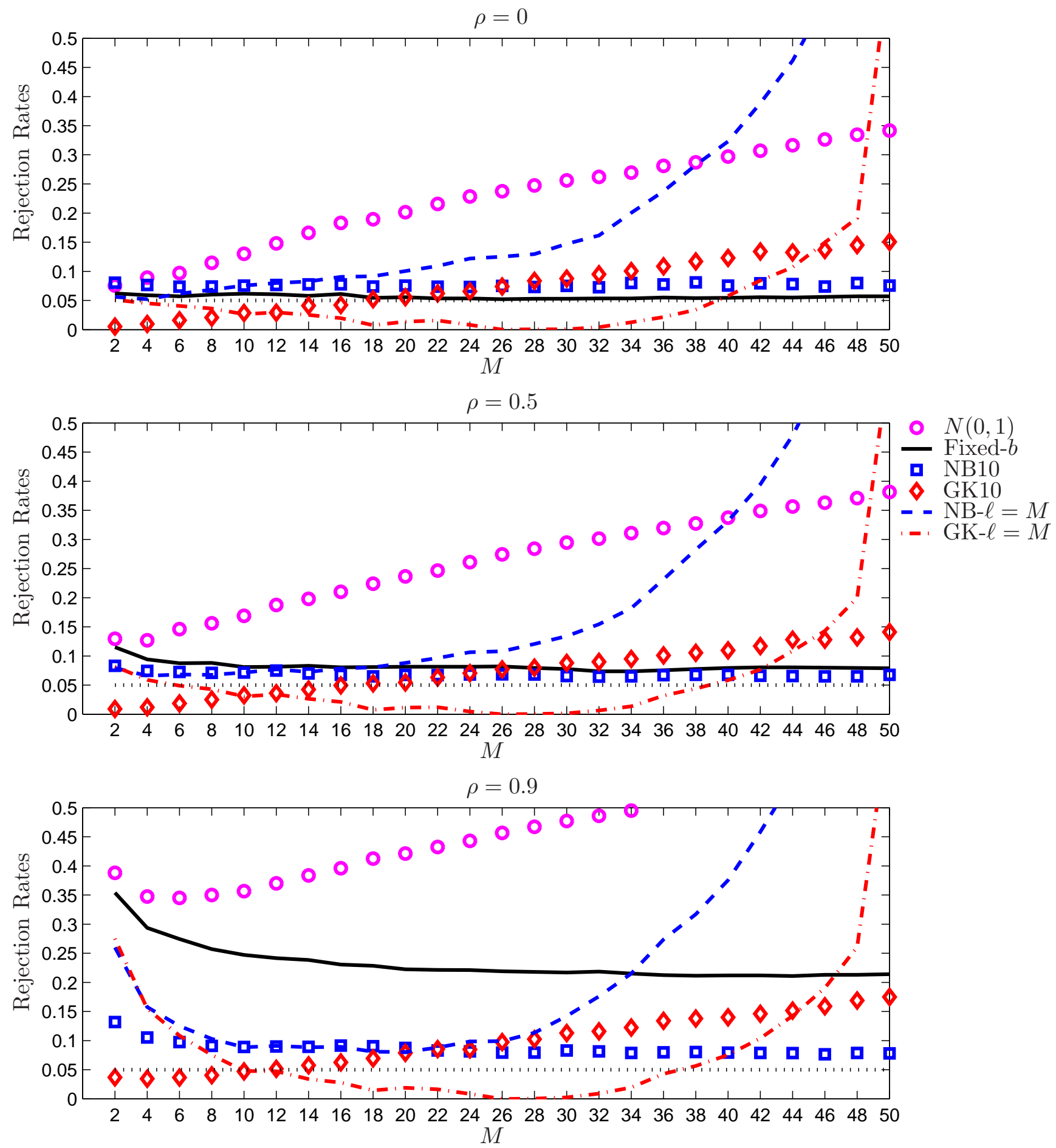

Figure 6: Empirical Null Rejection Probabilities, Linear Regression, Bartlett Kernel, $T=50$ 

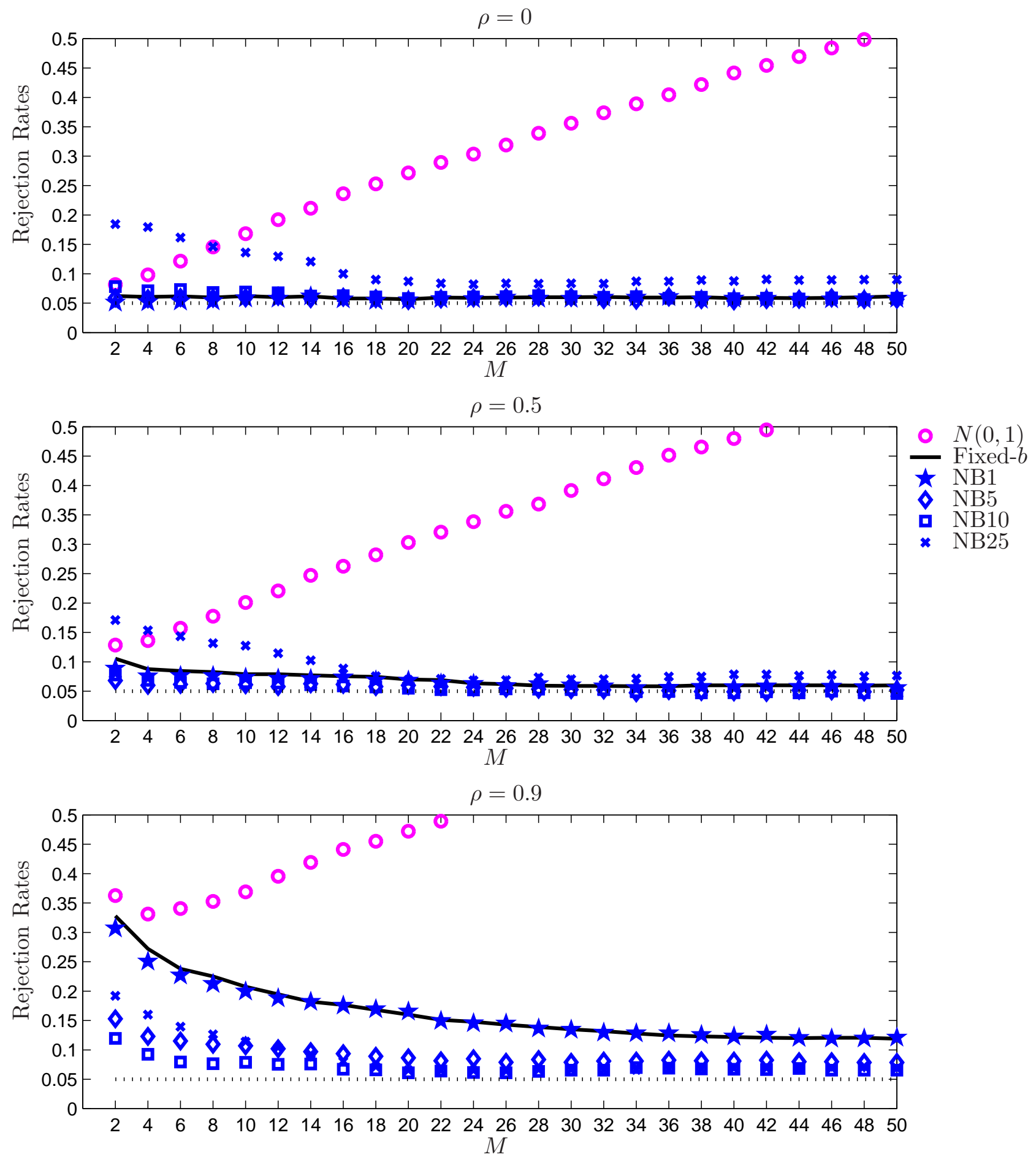

Figure 7: Empirical Null Rejection Probabilities, Linear Regression, QS Kernel, $T=50$ 

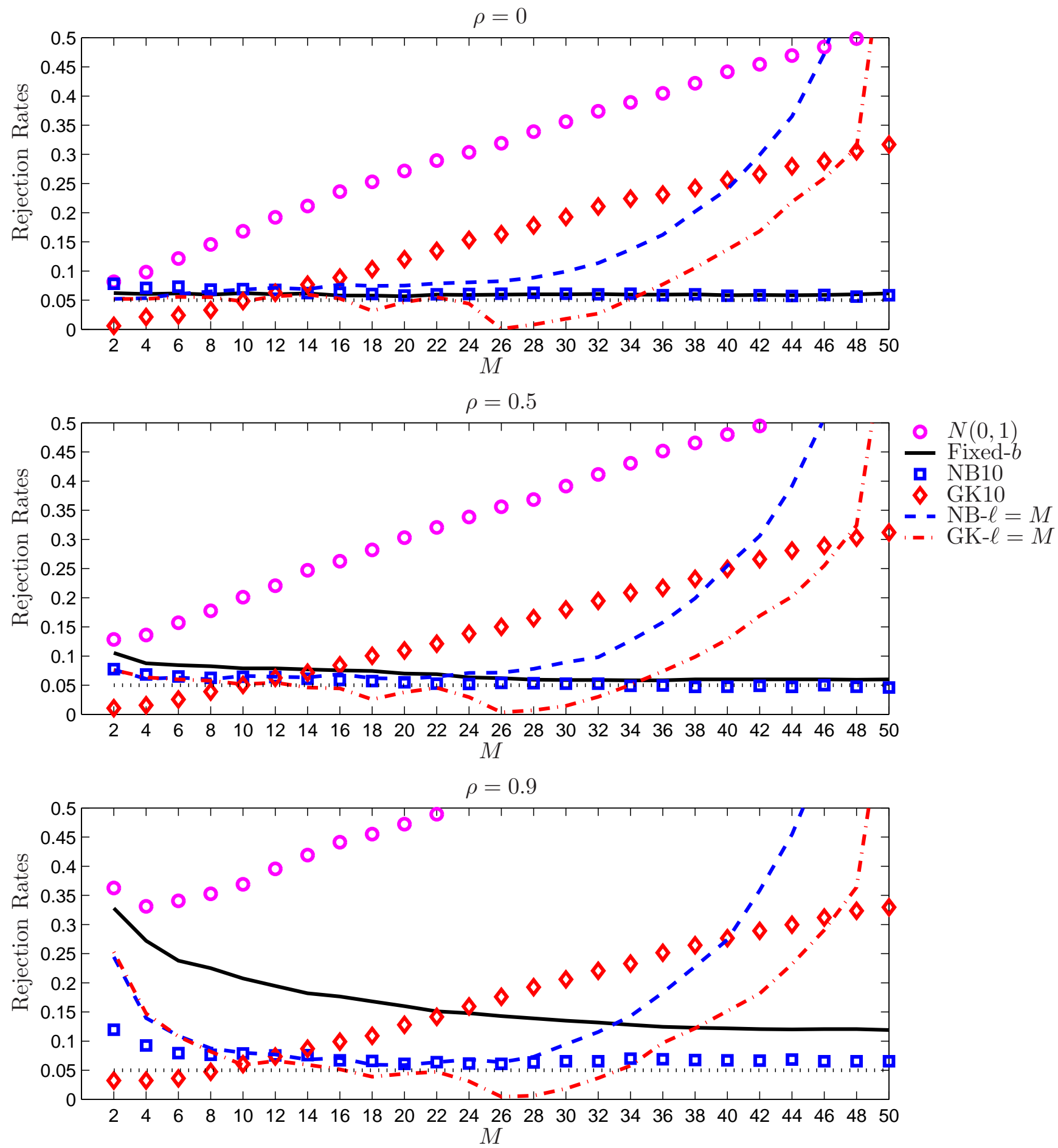

Figure 8: Empirical Null Rejection Probabilities, Linear Regression, QS Kernel, $T=50$ 